\title{
Sharp conditions for the linearization of finite elasticity
}

\author{
Edoardo Mainini $^{1}$ (D) $\cdot$ Danilo Percivale $^{1}$
}

Received: 21 January 2021 / Accepted: 13 June 2021 / Published online: 24 July 2021

(c) The Author(s) 2021

\begin{abstract}
We consider the topic of linearization of finite elasticity for pure traction problems. We characterize the variational limit for the approximating sequence of rescaled nonlinear elastic energies. We show that the limiting minimal value can be strictly lower than the minimal value of the standard linear elastic energy if a strict compatibility condition for external loads does not hold. The results are provided for both the compressible and the incompressible case.
\end{abstract}

Mathematics Subject Classification 49J45 $\cdot 74 \mathrm{~K} 30 \cdot 74 \mathrm{~K} 35 \cdot 74 \mathrm{R} 10$

\section{Introduction}

Let $\Omega \subset \mathbb{R}^{3}$ be the reference configuration of a hyperelastic body. If $\mathbf{y}: \Omega \rightarrow \mathbb{R}^{3}$ is the deformation field and $h>0$ is an adimensional parameter, we introduce the scaled global energy of the body, including the stored elastic energy and the work of external forces, by

$$
\mathcal{G}_{h}(\mathbf{y}):=h^{-2} \int_{\Omega} \mathcal{W}(\mathbf{x}, \nabla \mathbf{y}) d \mathbf{x}-h^{-1} \mathcal{L}(\mathbf{y}-\mathbf{i})
$$

Here, $\mathcal{W}: \Omega \times \mathbb{R}^{3 \times 3} \rightarrow[0,+\infty]$ is the strain energy density and $\mathbf{i}$ denotes the identity map. For every $\mathbf{x} \in \Omega$, the function $\mathcal{W}(\mathbf{x}, \cdot)$ is assumed to be frame indifferent and uniquely minimized at rotations with value 0 . It is also assumed to be $C^{2}$-smooth around rotations and to satisfy a suitable coercivity condition to be introduced later on. Moreover, the load functional $\mathcal{L}$ is defined by

$$
\mathcal{L}(\mathbf{v}):=\int_{\Omega} \mathbf{f} \cdot \mathbf{v} d \mathbf{x}+\int_{\partial \Omega} \mathbf{g} \cdot \mathbf{v} d \mathcal{H}^{2}(\mathbf{x}),
$$

Communicated by A. Malchiodi.

$\triangle$ Edoardo Mainini

mainini@dime.unige.it

Danilo Percivale

percivale@dime.unige.it

1 Dipartimento di Ingegneria meccanica, energetica, gestionale e dei trasporti, Università degli studi di Genova, Via all'Opera Pia, 15, 16145 Genova, Italy 
where $\mathbf{f}: \Omega \rightarrow \mathbb{R}^{3}$ is a volume force field, $\mathbf{g}: \partial \Omega \rightarrow \mathbb{R}^{3}$ is a surface force field, and $\mathcal{H}^{2}$ denotes the surface measure.

In a pure traction problem, in order to have uniform bounds from below for functionals $\mathcal{G}_{h}$ we have to assume that

$$
\mathcal{L}(\mathbf{y}-\mathbf{i}) \leq 0
$$

for every deformation $\mathbf{y}$ such that

$$
\int_{\Omega} \mathcal{W}(\mathbf{x}, \nabla \mathbf{y}) d \mathbf{x}=0
$$

Under our assumptions on $\mathcal{W},(1.2)$ holds true if and only if $\nabla \mathbf{y}$ is a constant rotation matrix, i.e., $\mathbf{y}(\mathbf{x})=\mathbf{R x}+\mathbf{c}$ for some $\mathbf{R} \in S O$ (3) and some $\mathbf{c} \in \mathbb{R}^{3}$, where $S O$ (3) denotes the special orthogonal group. Thus, we need to assume that

$$
\mathcal{L}((\mathbf{R}-\mathbf{I}) \mathbf{x}+\mathbf{c}) \leq 0
$$

for every $\mathbf{R} \in S O(3)$ and every $\mathbf{c} \in \mathbb{R}^{3}$, and by taking $\mathbf{R}=\mathbf{I}$ we get $\mathcal{L}(\mathbf{c}) \leq 0$ for every $\mathbf{c} \in \mathbb{R}^{3}$, that is, $\mathcal{L}(\mathbf{c})=0$ for every $\mathbf{c} \in \mathbb{R}^{3}$. Hence, (1.3) is equivalent to the following two conditions

$$
\begin{aligned}
\mathcal{L}(\mathbf{c})=0 & & \forall \mathbf{c} \in \mathbb{R}^{3}, \\
\mathcal{L}((\mathbf{R}-\mathbf{I}) \mathbf{x}) \leq 0 & & \forall \mathbf{R} \in S O(3) .
\end{aligned}
$$

We observe that indeed if $\mathbf{R} \in S O$ (3) exists such that $\mathcal{L}((\mathbf{R}-\mathbf{I}) \mathbf{x})>0$, then $\mathcal{G}_{h}$ is not uniformly bounded from below with respect to $h$, that is, inf $\mathcal{G}_{h} \rightarrow-\infty$ as $h \rightarrow 0$ (see also Remark 2.6 below). It is worth noting that (1.4) says that external loads have null resultant while it will be shown in Remark 2.1 that (1.5) implies they have null momentum (without being equivalent to the null momentum condition).

The choice of the scaling powers in (1.1) depends on the behavior of the elastic strain energy density and of the work expended by external loads for deformations which are close to a suitable rotation of the reference configuration, say $\mathbf{y}=\mathbf{R}(\mathbf{i}+h \mathbf{u})$, where $\mathbf{u}: \Omega \rightarrow \mathbb{R}^{3}$ and where $\mathbf{R}$ belongs to the following rotation kernel associated to $\mathcal{L}$ (that satisfies (1.4)-(1.5))

$$
\mathcal{S}_{\mathcal{L}}^{0}:=\{\mathbf{R} \in S O(3): \mathcal{L}((\mathbf{R}-\mathbf{I}) \mathbf{x})=0\} .
$$

Indeed, by frame indifference we obtain

$$
\mathcal{G}_{h}(\mathbf{y})=h^{-2} \int_{\Omega} \mathcal{W}(\mathbf{x}, \mathbf{I}+h \nabla \mathbf{u}(\mathbf{x})) d \mathbf{x}-\mathcal{L}(\mathbf{R u})-h^{-1} \mathcal{L}((\mathbf{R}-\mathbf{I}) \mathbf{x}),
$$

and if $\mathbf{R} \in \mathcal{S}_{\mathcal{L}}^{0}$, by a Taylor expansion of $\mathcal{W}(\mathbf{x}, \cdot)$ around the identity matrix we formally get for every fixed $\mathbf{u}$

$$
\lim _{h \rightarrow 0} \mathcal{G}_{h}(\mathbf{y})=\int_{\Omega} \mathcal{Q}(\mathbf{x}, \mathbb{E}(\mathbf{u})) d \mathbf{x}-\mathcal{L}(\mathbf{R u}),
$$

where $\mathbb{E}(\mathbf{u}):=\frac{1}{2}\left(\nabla \mathbf{u}^{T}+\nabla \mathbf{u}\right)$ and where we have introduced the quadratic form

$$
\mathcal{Q}(\mathbf{x}, \mathbf{F}):=\frac{1}{2} \mathbf{F}^{T} D^{2} \mathcal{W}(\mathbf{x}, \mathbf{I}) \mathbf{F}, \quad \mathbf{F} \in \mathbb{R}^{3 \times 3}, \quad \mathbf{x} \in \Omega .
$$

Therefore, it is natural to guess that the variational limit $\mathcal{G}$ of $\mathcal{G}_{h}$ as $h \rightarrow 0$ can be obtained from (1.8) through a minimization among all $\mathbf{R} \in \mathcal{S}_{\mathcal{L}}^{0}$, namely

$$
\mathcal{G}(\mathbf{u}):=\int_{\Omega} \mathcal{Q}(\mathbf{x}, \mathbb{E}(\mathbf{u})) d \mathbf{x}-\max _{\mathbf{R} \in \mathcal{S}_{\mathcal{L}}^{0}} \mathcal{L}(\mathbf{R u}) .
$$


We stress that, if $\mathbf{R}=\mathbf{I}$, this corresponds to the usual formal derivation of linearized elasticity. But for any other $\mathbf{R} \in \mathcal{S}_{\mathcal{L}}^{0}$ the work done by external loads for going from $\Omega$ to $\mathbf{R} \Omega$ is null, so that it might be energetically convenient to consider deformations near $\mathbf{R} \mathbf{x}$ rather than near the identity. On the other hand it is clear that if $\mathbf{R} \notin \mathcal{S}_{\mathcal{L}}^{0}$ this heuristic argument fails. Indeed, choosing $\mathbf{R} \in S O(3) \backslash \mathcal{S}_{\mathcal{L}}^{0}$ is not energetically convenient due to the behavior of the last term in the right hand side of (1.7) as $h \rightarrow 0$.

In the case that (1.4)-(1.5) hold and $\mathcal{S}_{\mathcal{L}}^{0} \equiv\{\mathbf{I}\}$, then (1.9) reduces to

$$
\mathcal{E}(\mathbf{u}):=\int_{\Omega} \mathcal{Q}(\mathbf{x}, \mathbb{E}(\mathbf{u})) d \mathbf{x}-\mathcal{L}(\mathbf{u}),
$$

i.e., to the form of the total potential energy that follows from the standard derivation of the linear theory for hyperelastic bodies, which is based on a linearization around the identity, see [7, Chapters IX-X].

It has been shown in [10] (see also [9]) that in this case

$$
\lim _{h \rightarrow 0}\left(\inf \mathcal{G}_{h}\right)=\min \mathcal{E}
$$

and if $\mathcal{G}_{h}\left(\mathbf{y}_{h}\right)-\inf \mathcal{G}_{h} \rightarrow 0$ as $h \rightarrow 0$ (i.e., if $\left(\mathbf{y}_{h}\right)$ is a sequence of quasi-minimizers of $\mathcal{G}_{h}$ ) then

$$
\mathbf{u}_{h}:=h^{-1}\left(\mathbf{y}_{h}-\mathbf{i}\right) \rightarrow \mathbf{u}_{0} \in \operatorname{argmin} \mathcal{E}
$$

in a suitable sense. In particular, $\mathbf{u}_{0}$ satisfies the equilibrium conditions

$$
\begin{cases}-\operatorname{div} \mathcal{Q}^{\prime}\left(\mathbf{x}, \mathbb{E}\left(\mathbf{u}_{0}\right)\right)=\mathbf{f} & \text { in } \Omega \\ \mathcal{Q}^{\prime}\left(\mathbf{x}, \mathbb{E}\left(\mathbf{u}_{0}\right)\right) \mathbf{n}=\mathbf{g} & \text { on } \partial \Omega,\end{cases}
$$

where $\mathcal{Q}^{\prime}(\mathbf{x}, \mathbf{F}):=D^{2} \mathcal{W}(\mathbf{x}, \mathbf{I}) \mathbf{F}$ and $\mathbf{n}$ is the outer unit normal to $\partial \Omega$. In [12] we have extended the results of [10] to incompressible elasticity. Indeed, it is shown in [12] that (1.10) and (1.11) hold true by substituting $\mathcal{G}_{h}$ with the scaled incompressible global energy $\mathcal{G}_{h}^{I}$, defined by replacing $\mathcal{W}$ with $\mathcal{W}^{I}$ in the right hand side of (1.1), where

$$
\mathcal{W}^{I}(\mathbf{x}, \mathbf{F}):= \begin{cases}\mathcal{W}(\mathbf{x}, \mathbf{F}) & \text { if } \operatorname{det} \mathbf{F}=1 \\ +\infty & \text { otherwise }\end{cases}
$$

and by substituting $\mathcal{E}$ with

$$
\mathcal{E}^{I}(\mathbf{v}):=\int_{\Omega} \mathcal{Q}^{I}(\mathbf{x}, \mathbb{E}(\mathbf{v}))-\mathcal{L}(\mathbf{v}),
$$

where

$$
\mathcal{Q}^{I}(\mathbf{x}, \mathbf{F}):= \begin{cases}\frac{1}{2} \mathbf{F}^{T} D^{2} \mathcal{W}(\mathbf{x}, \mathbf{I}) \mathbf{F} & \text { if } \operatorname{Tr} \mathbf{F}=0 \\ +\infty & \text { otherwise. }\end{cases}
$$

Roughly speaking, these results can be interpreted by saying that, if (1.4) holds along with the strict compatibility condition

$$
\mathcal{L}((\mathbf{R}-\mathbf{I}) \mathbf{x})<0 \quad \forall \mathbf{R} \in S O(3) \backslash\{\mathbf{I}\},
$$

then linear elasticity can be viewed as the variational limit of finite elasticity both in the compressible and in the incompressible case.

By assuming only (1.4) and (1.5), since $\mathcal{S}_{\mathcal{L}}^{0}$ need not be reduced to the identity matrix, a minimizer of functional (1.9) is not expected to satisfy (1.12) in general. On the other 
hand, we may ask if its energy level equals the minimal value of $\mathcal{E}$ : this fact is still an open question and it represents our main focus, along with the analogous comparison between optimal energy levels in the incompressible case.

A consequence of a recent result shown by Maor and Mora in [13, Theorem 5.3] is that if (1.4) and (1.5) hold along with a quadratic growth condition from below for $\mathcal{W}(\mathbf{x}, \cdot)$, then indeed

$$
\lim _{h \rightarrow 0}\left(\inf \mathcal{G}_{h}\right)=\min \mathcal{G} .
$$

In this paper we extend this result to the incompressible case and to more general coercivity assumptions on the strain energy density $\mathcal{W}$, but more than anything else we exhibit examples in which

$$
\min \mathcal{G}<\min \mathcal{E} \quad \text { and } \min \mathcal{G}^{I}<\min \mathcal{E}^{I},
$$

where $\mathcal{G}^{I}$ is defined by replacing $\mathcal{Q}$ with $\mathcal{Q}^{I}$ in (1.9). Surprisingly enough, this shows that, at least under the sole assumptions (1.4)-(1.5), the energy level of the minimizer of $\mathcal{E}$ does not necessarily provide the minimal value of the variational limit of the scaled finite elasticity functional $\mathcal{G}_{h}$. A gap between $\lim _{h \rightarrow 0}\left(\inf \mathcal{G}_{h}\right)\left(\right.$ resp. $\left.\lim _{h \rightarrow 0}\left(\inf \mathcal{G}_{h}^{I}\right)\right)$ and $\min \mathcal{E}$ (resp. $\min \mathcal{E}^{I}$ ) may appear.

In detail, by assuming the coercivity condition

$$
\mathcal{W}(\mathbf{x}, \mathbf{F}) \geq C g_{p}(d(\mathbf{F}, S O(3))) \quad \forall \mathbf{F} \in \mathbb{R}^{3 \times 3}
$$

for some $p \in(1,2]$, where

$$
g_{p}(t):= \begin{cases}t^{2} & \text { if } 0 \leq t \leq 1 \\ \frac{2 t^{p}}{p}-\frac{2}{p}+1 & \text { if } t \geq 1\end{cases}
$$

and $d(\cdot, S O(3))$ denotes the distance function from rotations, for the incompressible case we will prove the following result (see Theorem 2.5 below). If $\left(\mathbf{y}_{h}\right) \subset W^{1, p}\left(\Omega, \mathbb{R}^{3}\right)$ is a sequence of quasi-minimizers of $\mathcal{G}_{h}^{I}$, then by defining the generalized rescaled displacements $\mathbf{u}_{h}(\mathbf{x}):=h^{-1}\left(\mathbf{R}_{h}^{T} \mathbf{y}(\mathbf{x})-\mathbf{x}\right), \quad$ where $\quad \mathbf{R}_{h} \in \operatorname{argmin}\left\{\int_{\Omega} g_{p}\left(\left|\nabla \mathbf{y}_{h}-\mathbf{R}\right|\right) d \mathbf{x}: \mathbf{R} \in S O\right.$ (3) $\}$, there is a (not relabeled) subsequence such that

$$
\nabla \mathbf{u}_{h} \rightarrow \nabla \mathbf{u}_{*} \text { weakly in } L^{p}\left(\Omega, \mathbb{R}^{3 \times 3}\right) \quad \text { as } h \rightarrow 0,
$$

where $\mathbf{u}_{*} \in H^{1}\left(\Omega, \mathbb{R}^{3}\right)$ and $\mathbf{u}_{*}$ is a minimizer of $\mathcal{G}^{I}$ over $W^{1, p}\left(\Omega, \mathbb{R}^{3}\right)$. Moreover,

$$
\mathcal{G}_{h}^{I}\left(\mathbf{y}_{h}\right) \rightarrow \mathcal{G}^{I}\left(\mathbf{u}_{*}\right) \quad \text { and } \quad \inf _{W^{1, p}\left(\Omega, \mathbb{R}^{3}\right)} \mathcal{G}_{h}^{I} \rightarrow \min _{W^{1, p}\left(\Omega, \mathbb{R}^{3}\right)} \mathcal{G}^{I} \quad \text { as } h \rightarrow 0 .
$$

Here, the precise characterization of $\mathcal{G}^{I}$ is

$$
\mathcal{G}^{I}(\mathbf{u})= \begin{cases}\int_{\Omega} \mathcal{Q}^{I}(\mathbf{x}, \mathbb{E}(\mathbf{u})) d \mathbf{x}-\max _{\mathbf{R} \in \mathcal{S}_{\mathcal{L}}^{0}} \mathcal{L}(\mathbf{R u}) & \text { if } \mathbf{u} \in H_{\mathrm{div}}^{1}\left(\Omega, \mathbb{R}^{3}\right) \\ +\infty & \text { otherwise in } W^{1, p}\left(\Omega, \mathbb{R}^{3}\right),\end{cases}
$$

where $H_{\text {div }}^{1}\left(\Omega, \mathbb{R}^{3}\right)$ denotes the space of divergence-free $H^{1}\left(\Omega, \mathbb{R}^{3}\right)$ vector fields. Such a result improves the one in [12], as it allows to obtain the characterization of the limit energy even without the assumption $\mathcal{S}_{\mathcal{L}}^{0} \equiv\{\mathbf{I}\}$. 
It also generalizes a recent result of Jesenko and Schmidt [8] and reduces to it when $\mathcal{S}_{\mathcal{L}}^{0} \equiv S O$ (3). We will provide the same statement for the compressible case in Theorem 2.4, thus obtaining an analogous of [13, Theorem 5.3] for the case of the $p$-growth assumption (1.15) (see also Remark 2.12 below). On top of that, we will show in Theorem 2.7 that there are configurations and external loads such that the strict inequalities (1.14) hold, the minimization problems being cast on $W^{1, p}\left(\Omega, \mathbb{R}^{3}\right)$. We will end our analysis by remarking that (1.10) might be true even if (1.4)-(1.5) hold and $\mathcal{S}_{\mathcal{L}}^{0}$ is not reduced to the identity matrix: indeed, it is always possible to rotate the external forces in such a way that (1.10) holds for the problem with rotated forces, see Theorem 2.10.

Let us mention that our results about convergence of minimizers (Theorem 2.4 and Theorem 2.5) can also be adapted to higher space dimension, up to slight modifications to the proofs.

Let us finally mention that several other results about variational linearization of finite elasticity, including Dirichlet problems, incompressibility constraints or even theories for multiwell potentials are found in $[1-4,8,11,15]$.

\section{Plan of the paper}

In Sect. 2 we introduce the assumptions of the theory and state the main results. Section 3 collects some preliminary results. In Sect. 4 we provide the proof of the variational convergence results. Eventually, Sect. 5 delivers the main example with a limiting energy that is below the minimal value of the standard linearized elasticity functional.

\section{Main results}

We introduce the setting for compressible and incompressible elasticity, then we state the main results. In the following, the reference configuration $\Omega$ is always assumed to be a bounded open connected Lipschitz set in $\mathbb{R}^{3}$.

As basic notation, $\mathbb{R}^{3 \times 3}$ is the set of $3 \times 3$ real matrices, endowed with the Euclidean norm $|\mathbf{F}|=\sqrt{\mathbf{F}^{T} \mathbf{F}} \cdot \mathbb{R}_{\text {sym }}^{3 \times 3}$ (resp. $\mathbb{R}_{\text {skew }}^{3 \times 3}$ ) denotes the subset of symmetric (resp. skew-symmetric) matrices. For every $\mathbf{F} \in \mathbb{R}^{3 \times 3}$ we define $\operatorname{sym} \mathbf{F}:=\frac{1}{2}\left(\mathbf{F}+\mathbf{F}^{T}\right)$ and skew $\mathbf{F}:=\frac{1}{2}\left(\mathbf{F}-\mathbf{F}^{T}\right)$. By $S O$ (3) we denote the special orthogonal group and for every $\mathbf{R} \in S O$ (3) there exist $\vartheta \in \mathbb{R}$ and $\mathbf{W} \in \mathbb{R}_{\text {skew }}^{3 \times 3}$, such that $|\mathbf{W}|^{2}=\left|\mathbf{W}^{2}\right|^{2}=2$ and such that the following Euler-Rodrigues representation formula holds

$$
\mathbf{R}=\mathbf{I}+\sin \vartheta \mathbf{W}+(1-\cos \vartheta) \mathbf{W}^{2}
$$

\section{Assumptions on the elastic energy density}

We let $\mathcal{W}: \Omega \times \mathbb{R}^{3 \times 3} \rightarrow[0,+\infty]$ be $\mathcal{L}^{3} \times \mathcal{B}^{9}$ - measurable satisfying the following assumptions, see also $[2,11]$ :

$$
\mathcal{W}(\mathbf{x}, \mathbf{R F})=\mathcal{W}(\mathbf{x}, \mathbf{F}) \quad \forall \mathbf{R} \in S O(3) \quad \forall \mathbf{F} \in \mathbb{R}^{3 \times 3}, \quad \text { for a.e. } \mathbf{x} \in \Omega,
$$

$$
\min \mathcal{W}=\mathcal{W}(\mathbf{x}, \mathbf{I})=0 \text { for a.e. } \mathbf{x} \in \Omega
$$


Concerning the regularity of $\mathcal{W}$, we assume that there exist an open neighborhood $\mathcal{U}$ of $S O$ (3) in $\mathbb{R}^{3 \times 3}$, an increasing function $\omega: \mathbb{R}_{+} \rightarrow \mathbb{R}$ satisfying $\lim _{t \rightarrow 0^{+}} \omega(t)=0$ and a constant $K>0$ such that for a.e. $\mathbf{x} \in \Omega$

$$
\begin{aligned}
& \mathcal{W}(\mathbf{x}, \cdot) \in C^{2}(\mathcal{U}), \quad\left|D^{2} \mathcal{W}(\mathbf{x}, \mathbf{I})\right| \leq K \text { and } \\
& \left|D^{2} \mathcal{W}(\mathbf{x}, \mathbf{F})-D^{2} \mathcal{W}(\mathbf{x}, \mathbf{G})\right| \leq \omega(|\mathbf{F}-\mathbf{G}|) \quad \forall \mathbf{F}, \mathbf{G} \in \mathcal{U}
\end{aligned}
$$

We assume in addition the following growth property from below: there exist $C>0$ and $p \in(1,2]$ such that for a.e. $\mathbf{x} \in \Omega$

$$
\mathcal{W}(\mathbf{x}, \mathbf{F}) \geq C g_{p}(d(\mathbf{F}, S O(3))) \quad \forall \mathbf{F} \in \mathbb{R}^{3 \times 3},
$$

where $g_{p}:[0,+\infty) \rightarrow \mathbb{R}$ is the strictly convex function defined by (1.16). We notice that a standard application of the Hölder inequality shows that for every $\eta \in L^{p}(\Omega)$ and every $h \in(0,1)$

$$
\begin{aligned}
h^{-2} \int_{\Omega} g_{p}(h|\eta|) d t & \geq \int_{|\eta| \leq h^{-1}}|\eta|^{2} d t+h^{p-2} \int_{|\eta| \geq h^{-1}}|\eta|^{p} d t \\
& \geq \frac{2}{p} \int_{|\eta| \leq h^{-1}}|\eta|^{p} d t+h^{p-2} \int_{|\eta| \geq h^{-1}}|\eta|^{p} d t-\frac{2-p}{p}|\Omega| \\
& \geq \int_{\Omega}|\eta|^{p} d t-\frac{2-p}{p}|\Omega| .
\end{aligned}
$$

In order to consider incompressible elasticity models, starting from a function $\mathcal{W}$ as above we also introduce the incompressible strain energy density by letting, for a.e. $\mathbf{x} \in \Omega$,

$$
\mathcal{W}^{I}(\mathbf{x}, \mathbf{F}):= \begin{cases}\mathcal{W}(\mathbf{x}, \mathbf{F}) & \text { if } \operatorname{det} \mathbf{F}=1 \\ +\infty & \text { otherwise }\end{cases}
$$

\section{Assumptions on the external forces}

We introduce a body force field $\mathbf{f} \in L^{\frac{3 p}{4 p-3}}\left(\Omega, \mathbb{R}^{3}\right)$ and a surface force field $\mathbf{g} \in$ $L^{\frac{2 p}{3 p-3}}\left(\partial \Omega, \mathbb{R}^{3}\right)$, where $p$ is such that $(\mathcal{W} \mathbf{4})$ holds. From here on, $\mathbf{f}$ and $\mathbf{g}$ will always be understood to satisfy such summability assumptions. The load functional is the following linear functional

$$
\mathcal{L}(\mathbf{v}):=\int_{\Omega} \mathbf{f} \cdot \mathbf{v} d \mathbf{x}+\int_{\partial \Omega} \mathbf{g} \cdot \mathbf{v} d \mathcal{H}^{2}(\mathbf{x}), \quad \mathbf{v} \in W^{1, p}\left(\Omega, \mathbb{R}^{3}\right) .
$$

We note that since $\Omega$ is a bounded Lipschitz domain, the Sobolev embedding $W^{1, p}\left(\Omega, \mathbb{R}^{3}\right) \hookrightarrow L^{\frac{3 p}{3-p}}\left(\Omega, \mathbb{R}^{3}\right)$ and the Sobolev trace embedding $W^{1, p}\left(\Omega, \mathbb{R}^{3}\right) \hookrightarrow$ $L^{\frac{2 p}{3-p}}\left(\partial \Omega, \mathbb{R}^{3}\right)$ imply that $\mathcal{L}$ is a bounded functional over $W^{1, p}\left(\Omega, \mathbb{R}^{3}\right)$.

We assume that external loads have null resultant

$$
\mathcal{L}(\mathbf{c})=0 \quad \forall \mathbf{c} \in \mathbb{R}^{3}
$$

and that they satisfy the following weak compatibility condition

$$
\mathcal{L}((\mathbf{R}-\mathbf{I}) \mathbf{x}) \leq 0 \quad \forall \mathbf{R} \in S O(3) .
$$

A crucial object in our results is the rotation kernel $\mathcal{S}_{\mathcal{L}}^{0}$ associated to a functional $\mathcal{L}$ satisfying the above assumptions, which is the set defined by (1.6). Such a kernel includes at least the identity matrix and represents the set of rotations that realize equality in $(\mathcal{L} 2)$. 
Remark 2.1 Thanks to the the Euler-Rodrigues representation formula for rotations (2.1), it is readily seen that $(\mathcal{L} 2)$ may be rewritten as

$$
h_{\mathbf{W}}(\theta):=\mathcal{L}(\mathbf{W} \mathbf{x}) \sin \theta+(1-\cos \theta) \mathcal{L}\left(\mathbf{W}^{2} \mathbf{x}\right) \leq 0
$$

for every $\theta \in[0,2 \pi]$ and for every $\mathbf{W} \in \mathbb{R}_{\text {skew }}^{3 \times 3}$ with $|\mathbf{W}|=2$. Since $h_{\mathbf{W}}(0)=h_{\mathbf{W}}(2 \pi)=0$, then

$$
0 \leq h_{\mathbf{W}}^{\prime}(2 \pi)=\mathcal{L}(\mathbf{W} \mathbf{x})=h_{\mathbf{W}}^{\prime}(0) \leq 0,
$$

that is, by linearity of $\mathcal{L}$,

$$
\mathcal{L}(\mathbf{W x})=0 \quad \forall \mathbf{W} \in \mathbb{R}_{\text {skew }}^{3 \times 3},
$$

and so if $(\mathcal{L} 2)$ holds then external loads have null momentum. Therefore, $(\mathcal{L} 2)$ is equivalent to

$$
\mathcal{L}(\mathbf{W} \mathbf{x})=0, \quad \mathcal{L}\left(\mathbf{W}^{2} \mathbf{x}\right) \leq 0 \quad \forall \mathbf{W} \in \mathbb{R}_{\text {skew }}^{3 \times 3},
$$

and we mention that formulation (2.4) of the compatibility condition (with strict inequality) is the one appearing in $[9,10,12]$. On the other hand it is worth noting that the null momentum condition does not imply the second relation in (2.4). Indeed, let $\mathbf{f}(\mathbf{x})=-\mathbf{x}, \mathbf{g} \equiv 0$ and let $\Omega$ be the open unit ball in $\mathbb{R}^{3}$. Then

$$
\mathcal{L}\left(\mathbf{W}^{2} \mathbf{x}\right)=\int_{\Omega}|\mathbf{W} \mathbf{x}|^{2} d \mathbf{x}>0
$$

for every $\mathbf{W} \in \mathbb{R}_{\text {skew }}^{3 \times 3}, \mathbf{W} \neq \equiv 0$, despite that external loads have null resultant and null momentum.

Remark 2.2 The characterization (2.4) of $(\mathcal{L} 2)$ and Euler-Rodrigues formula entail

$$
\mathcal{S}_{\mathcal{L}}^{0}=\{\mathbf{R} \in S O(3): \mathcal{L}((\mathbf{R}-\mathbf{I}) \mathbf{x})=0\}=\left\{e^{\mathbf{W}}: \mathbf{W} \in \mathcal{X}_{\mathcal{L}}^{0}\right\}
$$

where

$$
\mathcal{X}_{\mathcal{L}}^{0}:=\left\{\mathbf{W} \in \mathbb{R}_{\text {skew }}^{3 \times 3}: \mathcal{L}(\mathbf{W} \mathbf{x})=\mathcal{L}\left(\mathbf{W}^{2} \mathbf{x}\right)=0\right\} .
$$

Therefore, we have $\mathbf{R} \in \mathcal{S}_{\mathcal{L}}^{0} \Rightarrow \mathbf{R}^{T} \in \mathcal{S}_{\mathcal{L}}^{0}$, because

$$
\mathcal{L}\left(\left(\mathbf{R}^{T}-\mathbf{I}\right) \mathbf{x}\right)=\mathcal{L}\left(\left(\mathbf{R}^{T}-\mathbf{R}\right) \mathbf{x}\right)+\mathcal{L}((\mathbf{R}-\mathbf{I}) \mathbf{x})=0
$$

holds true since $\mathbf{R}^{T}-\mathbf{R}$ is skew-symmetric. Moreover, if $\mathbf{W}_{i} \in \mathcal{X}_{\mathcal{L}}^{0}, i=1,2$, then by $(\mathcal{L} 2)$

$$
\begin{aligned}
0 & \geq \mathcal{L}\left(\left(\mathbf{W}_{1} \pm \mathbf{W}_{2}\right)^{2} \mathbf{x}\right)=\mathcal{L}\left(\mathbf{W}_{1}^{2} \mathbf{x}\right)+\mathcal{L}\left(\mathbf{W}_{2}^{2} \mathbf{x}\right) \pm \mathcal{L}\left(\left(\mathbf{W}_{1} \mathbf{W}_{2}+\mathbf{W}_{2} \mathbf{W}_{1}\right) \mathbf{x}\right) \\
& = \pm \mathcal{L}\left(\left(\mathbf{W}_{1} \mathbf{W}_{2}+\mathbf{W}_{2} \mathbf{W}_{1}\right) \mathbf{x}\right),
\end{aligned}
$$

that is, $\mathbf{W}_{1} \pm \mathbf{W}_{2} \in \mathcal{X}_{\mathcal{L}}^{0}$, hence $e^{\mathbf{W}_{1} \pm \mathbf{W}_{2}} \in \mathcal{S}_{\mathcal{L}}^{0}$. By recalling that $\mathbf{I} \in \mathcal{S}_{\mathcal{L}}^{0}$, we conclude that $\mathcal{S}_{\mathcal{L}}^{0}$ is a subgroup of $S O(3)$. A more detailed characterization of the set $\mathcal{S}_{\mathcal{L}}^{0}$ can be obtained from the results in [13].

\section{Energy functionals}

The rescaled finite elasticity functionals $\mathcal{G}_{h}: W^{1, p}\left(\Omega, \mathbb{R}^{3}\right) \rightarrow \mathbb{R} \cup\{+\infty\}$ are defined by (1.1) 
and the limit energy functional $\mathcal{G}: W^{1, p}\left(\Omega, \mathbb{R}^{3}\right) \rightarrow \mathbb{R} \cup\{+\infty\}$ is defined as

$$
\mathcal{G}(\mathbf{u}):= \begin{cases}\int_{\Omega} \mathcal{Q}(\mathbf{x}, \mathbb{E}(\mathbf{u})) d \mathbf{x}-\mathcal{L}(\mathbf{u})-\max _{\mathbf{R} \in \mathcal{S}_{\mathcal{L}}^{0}} \mathcal{L}((\mathbf{R}-\mathbf{I}) \mathbf{u}) & \text { if } \mathbf{u} \in H^{1}\left(\Omega, \mathbb{R}^{3}\right) \\ +\infty & \text { otherwise in } W^{1, p}\left(\Omega, \mathbb{R}^{3}\right),\end{cases}
$$

where $\mathcal{Q}(\mathbf{x}, \mathbf{F}):=\frac{1}{2} \mathbf{F}^{T} D^{2} \mathcal{W}(\mathbf{x}, \mathbf{I}) \mathbf{F}$. By introducing the standard functional of linearized elasticity $\mathcal{E}: W^{1, p}\left(\Omega, \mathbb{R}^{3}\right) \rightarrow \mathbb{R} \cup\{+\infty\}$, namely

$$
\mathcal{E}(\mathbf{u}):= \begin{cases}\int_{\Omega} \mathcal{Q}(\mathbf{x}, \mathbb{E}(\mathbf{u})) d \mathbf{x}-\mathcal{L}(\mathbf{u}) & \text { if } \mathbf{u} \in H^{1}\left(\Omega, \mathbb{R}^{3}\right) \\ +\infty & \text { otherwise in } W^{1, p}\left(\Omega, \mathbb{R}^{3}\right),\end{cases}
$$

we immediately see that $\mathcal{G} \leq \mathcal{E}$, since $\mathbf{I} \in \mathcal{S}_{\mathcal{L}}^{0}$ and $\mathcal{L}(\mathbf{0})=0$. It is well-known that $\mathcal{E}$ admits a unique minimizer up to infinitesimal rigid displacements (i.e., up to the addition of a displacements field $\mathbf{v}$ such that $\mathbb{E}(\mathbf{v})=0)$. Since the optimization problem in the definition of $\mathcal{G}$ is among rotations in $\mathcal{S}_{\mathcal{L}}^{0}$, it is not difficult to check that $\mathcal{G}$ is invariant under the addition of infinitesimal rigid displacements, i.e., $\mathcal{G}(\mathbf{u}+\mathbf{v})=\mathcal{G}(\mathbf{u})$ whenever $\mathbb{E}(\mathbf{v}) \equiv 0$. On the other hand, in general minimizers of $\mathcal{G}$ are not unique up to infinitesimal rigid displacements (see Proposition 5.2 later on).

When considering incompressible elasticity, the functional $\mathcal{G}_{h}^{I}: W^{1, p}\left(\Omega, \mathbb{R}^{3}\right) \rightarrow \mathbb{R} \cup$ $\{+\infty\}$, representing the scaled total energy, is defined by

$$
\mathcal{G}_{h}^{I}(\mathbf{y}):=h^{-2} \int_{\Omega} \mathcal{W}^{I}(\mathbf{x}, \nabla \mathbf{y}) d \mathbf{x}-h^{-1} \mathcal{L}(\mathbf{y}-\mathbf{i}),
$$

while the limit functional $\mathcal{G}^{I}: W^{1, p}\left(\Omega, \mathbb{R}^{3}\right) \rightarrow \mathbb{R} \cup\{+\infty\}$ is defined by

$$
\mathcal{G}^{I}(\mathbf{u}):= \begin{cases}\int_{\Omega} \mathcal{Q}^{I}(\mathbf{x}, \mathbb{E}(\mathbf{u})) d \mathbf{x}-\mathcal{L}(\mathbf{u})-\max _{\mathbf{R} \in \mathcal{S}_{\mathcal{L}}^{0}} \mathcal{L}((\mathbf{R}-\mathbf{I}) \mathbf{u}) & \text { if } \mathbf{u} \in H^{1}\left(\Omega, \mathbb{R}^{3}\right) \\ +\infty & \text { otherwise in } W^{1, p}\left(\Omega, \mathbb{R}^{3}\right),\end{cases}
$$

where $\mathcal{Q}^{I}$ is defined by (1.13). We also introduce the functional of incompressible linearized elasticity $\mathcal{E}^{I}: W^{1, p}\left(\Omega, \mathbb{R}^{3}\right) \rightarrow \mathbb{R} \cup\{+\infty\}$, namely

$$
\mathcal{E}^{I}(\mathbf{u}):= \begin{cases}\int_{\Omega} \mathcal{Q}^{I}(\mathbf{x}, \mathbb{E}(\mathbf{u})) d \mathbf{x}-\mathcal{L}(\mathbf{u}) & \text { if } \mathbf{u} \in H^{1}\left(\Omega, \mathbb{R}^{3}\right) \\ +\infty & \text { otherwise in } W^{1, p}\left(\Omega, \mathbb{R}^{3}\right),\end{cases}
$$

and again $\mathcal{G}^{I} \leq \mathcal{E}^{I}$. Functional $\mathcal{E}^{I}$ admits a unique minimizer up to infinitesimal rigid displacements.

Before moving to the statement of the main results, we introduce a couple of definitions. For every $\mathbf{y} \in W^{1, p}\left(\Omega, \mathbb{R}^{3}\right)$ we define

$$
\mathcal{A}_{p}(\mathbf{y}):=\operatorname{argmin}\left\{\int_{\Omega} g_{p}(|\nabla \mathbf{y}-\mathbf{R}|) d \mathbf{x}: \mathbf{R} \in S O(3)\right\} .
$$

Moreover, we may combine this definition with the rigidity inequality by Friesecke, James and Müller [5], in the general form appearing in [2,6], to get the following estimate. There exists 
a constant $C_{p}=C_{p}(\Omega)>0$ such that for every $\mathbf{y} \in W^{1, p}\left(\Omega, \mathbb{R}^{3}\right)$ and every $\mathbf{R} \in \mathcal{A}_{p}(\mathbf{y})$

$$
\int_{\Omega} g_{p}(|\nabla \mathbf{y}-\mathbf{R}|) d \mathbf{x} \leq C_{p} \int_{\Omega} g_{p}(d(\nabla \mathbf{y}, S O(3))) d \mathbf{x},
$$

where $d(\mathbf{F}, S O(3)):=\inf \{|\mathbf{F}-\mathbf{R}|: \mathbf{R} \in S O(3)\}$.

Moreover, we introduce the following

Definition 2.3 Given a vanishing sequence $\left(h_{j}\right)_{j \in \mathbb{N}} \subset(0,1)$, we say that $\left(\mathbf{y}_{j}\right)_{j \in \mathbb{N}} \subset$ $W^{1, p}\left(\Omega, \mathbb{R}^{3}\right)$ is a sequence of quasi-minimizers of $\mathcal{G}_{h_{j}}$ if

$$
\lim _{j \rightarrow+\infty}\left(\mathcal{G}_{h_{j}}\left(\mathbf{y}_{j}\right)-\inf _{W^{1, p}\left(\Omega, \mathbb{R}^{3}\right)} \mathcal{G}_{h_{j}}\right)=0 .
$$

Sequences of quasi-minimizers of $\mathcal{G}_{h_{j}}^{I}$ are defined in the same way.

\section{Convergence results}

We are ready for the statement of the convergence result in the compressible case.

Theorem 2.4 Assume $(\mathcal{L} 1),(\mathcal{L} 2),(\mathcal{W} 1),(\mathcal{W} 2),(\mathcal{W} 3),(\mathcal{W} 4)$. Let $\left(h_{j}\right)_{j \in \mathbb{N}} \subset(0,1)$ be a vanishing sequence. Then we have

$$
\inf _{W^{1, p}\left(\Omega, \mathbb{R}^{3}\right)} \mathcal{G}_{h_{j}} \in \mathbb{R} \quad \text { for any } j \in \mathbb{N} .
$$

Moreover, if $\left(\mathbf{y}_{j}\right)_{j \in \mathbb{N}} \subset W^{1, p}\left(\Omega, \mathbb{R}^{3}\right)$ is a sequence of quasi-minimizers of $\mathcal{G}_{h_{j}}$, and if $\mathbf{R}_{j} \in \mathcal{A}_{p}\left(\mathbf{y}_{j}\right)$ for any $j \in \mathbb{N}$, then by defining

$$
\mathbf{u}_{j}(\mathbf{x}):=h_{j}^{-1}\left(\mathbf{R}_{j}^{T} \mathbf{y}(\mathbf{x})-\mathbf{x}\right)
$$

there is a (not relabeled) subsequence such that

$$
\nabla \mathbf{u}_{j} \rightarrow \nabla \mathbf{u}_{*} \text { weakly in } L^{p}\left(\Omega, \mathbb{R}^{3 \times 3}\right) \quad \text { as } j \rightarrow+\infty,
$$

where $\mathbf{u}_{*} \in H^{1}\left(\Omega, \mathbb{R}^{3}\right)$ is a minimizer of $\mathcal{G}$ over $W^{1, p}\left(\Omega, \mathbb{R}^{3}\right)$, and

$$
\mathcal{G}_{h_{j}}\left(\mathbf{y}_{j}\right) \rightarrow \mathcal{G}\left(\mathbf{u}_{*}\right), \quad \inf _{W^{1, p}\left(\Omega, \mathbb{R}^{3}\right)} \mathcal{G}_{h_{j}} \rightarrow \min _{W^{1, p}\left(\Omega, \mathbb{R}^{3}\right)} \mathcal{G} \quad \text { as } j \rightarrow+\infty .
$$

The same statement holds in the incompressible case. Indeed, under an additional assumption on $\partial \Omega$, which is needed in the proof when invoking a technical approximation lemma from [12], we have the following result.

Theorem 2.5 Assume that $\partial \Omega$ has a finite number of connected components. Assume $(\mathcal{L} 1)$, $(\mathcal{L} 2),(\mathcal{W} 1),(\mathcal{W} 2),(\mathcal{W} 3),(\mathcal{W} 4)$. Let $\left(h_{j}\right)_{j \in \mathbb{N}} \subset(0,1)$ be a vanishing sequence. Then we have

$$
\inf _{W^{1, p}\left(\Omega, \mathbb{R}^{3}\right)} \mathcal{G}_{h_{j}}^{I} \in \mathbb{R} \quad \text { for any } j \in \mathbb{N} .
$$

Moreover, if $\left(\mathbf{y}_{j}\right)_{j \in \mathbb{N}} \subset W^{1, p}\left(\Omega, \mathbb{R}^{3}\right)$ is a sequence of quasi-minimizers of $\mathcal{G}_{h_{j}}^{I}$, and if $\mathbf{R}_{j} \in \mathcal{A}_{p}\left(\mathbf{y}_{j}\right)$ for any $j \in \mathbb{N}$, then by defining

$$
\mathbf{u}_{j}(\mathbf{x}):=h_{j}^{-1}\left(\mathbf{R}_{j}^{T} \mathbf{y}(\mathbf{x})-\mathbf{x}\right)
$$

there is a (not relabeled) subsequence such that

$$
\nabla \mathbf{u}_{j} \rightarrow \nabla \mathbf{u}_{*} \text { weakly in } L^{p}\left(\Omega, \mathbb{R}^{3 \times 3}\right) \quad \text { as } j \rightarrow+\infty,
$$


where $\mathbf{u}_{*} \in H^{1}\left(\Omega, \mathbb{R}^{3}\right)$ is a minimizer of $\mathcal{G}^{I}$ over $W^{1, p}\left(\Omega, \mathbb{R}^{3}\right)$, and

$$
\mathcal{G}_{h_{j}}^{I}\left(\mathbf{y}_{j}\right) \rightarrow \mathcal{G}^{I}\left(\mathbf{u}_{*}\right), \quad \inf _{W^{1, p}\left(\Omega, \mathbb{R}^{3}\right)} \mathcal{G}_{h_{j}}^{I} \rightarrow \min _{W^{1, p}\left(\Omega, \mathbb{R}^{3}\right)} \mathcal{G}^{I} \quad \text { as } j \rightarrow+\infty .
$$

Remark 2.6 Inequality in $(\mathcal{L} 2)$ can never be reversed. Indeed let us assume $(\mathcal{L} 1),(\mathcal{W} \mathbf{1})$, $(\mathcal{W} 2),(\mathcal{W} 3),(\mathcal{W} 4)$ and that there exists $\mathbf{R}_{*} \in S O(3)$ such that

$$
\mathcal{L}\left(\left(\mathbf{R}^{*}-\mathbf{I}\right) \mathbf{x}\right)>0 .
$$

Then it is readily seen that by setting $\mathbf{y}_{j}^{*}(\mathbf{x})=\mathbf{R}^{*} \mathbf{x}$ we get

$$
\mathcal{G}_{h_{j}}\left(\mathbf{y}_{j}^{*}\right)=-h_{j}^{-1} \mathcal{L}\left(\left(\mathbf{R}^{*}-\mathbf{I}\right) \mathbf{x}\right) \rightarrow-\infty \quad \text { as } j \rightarrow+\infty .
$$

For instance, we notice that if the body is subject to a uniform boundary compressive force field then (2.7) occurs for every $\mathbf{R} \in S O(3), \mathbf{R} \neq \mathbf{I}$. Indeed, if $\mathbf{n}$ denotes the outer unit normal vector to $\partial \Omega$, and we choose $\mathbf{g}=\lambda \mathbf{n}$ with $\lambda<0$ and $\mathbf{f} \equiv 0$, then

$$
\int_{\partial \Omega} \mathbf{g} \cdot(\mathbf{R}-\mathbf{I}) \mathbf{x} d \mathcal{H}^{2}(\mathbf{x})=\lambda(\operatorname{Tr}(\mathbf{R}-\mathbf{I}))|\Omega|>0 \quad \forall \mathbf{R} \in S O(3), \mathbf{R} \neq \mathbf{I} .
$$

\section{The gap with linear elasticity}

By summarizing, if $(\mathcal{L} \mathbf{1}),(\mathcal{L} 2),(\mathcal{W} \mathbf{1}),(\mathcal{W} \mathbf{2}),(\mathcal{W} \mathbf{3}),(\mathcal{W} \mathbf{4})$ are satisfied and $\mathcal{S}_{\mathcal{L}}^{0} \equiv\{\mathbf{I}\}$ then functionals $\mathcal{G}^{I}$ and $\mathcal{G}$ are the classical functionals of linear incompressible and compressible elasticity, respectively.

On the other hand if (2.7) occurs then by Remark 2.6 no convergence result is possible. In all other cases, although a full description of the limit functionals is given by means of $\mathcal{G}$ and $\mathcal{G}^{I}$, it is not a priori clear if their minimal values coincide with the minimal values of linear (compressible or incompressible) elasticity. In order to complete the picture, we will show that there exist configurations and external forces satisfying the assumptions of Theorem 2.4 and Theorem 2.5 (and such that $\mathcal{S}_{\mathcal{L}}^{0}$ is not reduced to the identity matrix) for which

$$
\min _{W^{1, p}\left(\Omega, \mathbb{R}^{3}\right)} \mathcal{G}<\min _{W^{1, p}\left(\Omega, \mathbb{R}^{3}\right)} \mathcal{E}
$$

and

$$
\min _{W^{1, p}\left(\Omega, \mathbb{R}^{3}\right)} \mathcal{G}^{I}<\min _{W^{1, p}\left(\Omega, \mathbb{R}^{3}\right)} \mathcal{E}^{I},
$$

Therefore, in such case, given any vanishing sequence $\left(h_{j}\right)_{j \in \mathbb{N}} \subset(0,1)$ and any sequence $\left(\mathbf{y}_{j}\right)_{j \in \mathbb{N}} \subset W^{1, p}\left(\Omega, \mathbb{R}^{3}\right)$ of quasi-minimizers of $\mathcal{G}_{h_{j}}$ (resp. $\mathcal{G}_{h_{j}}^{I}$ ) there is no subsequence such that $\mathcal{G}_{h_{j}}\left(\mathbf{y}_{j}\right) \rightarrow \min \mathcal{E}\left(\operatorname{resp} . \mathcal{G}_{h_{j}}^{I}\left(\mathbf{y}_{j}\right) \rightarrow \min \mathcal{E}^{I}\right)$. This shows that the minimal value of the usual functional of linearized elasticity $\mathcal{E}$ (resp. $\mathcal{E}^{I}$ ) need not be the correct approximation of inf $\mathcal{G}_{h}$ (resp. $\inf \mathcal{G}_{h}^{I}$ ) in the regime of small $h$.

We are going to analyze in detail an example of validity of (2.8) and (2.9). The setting for such an example is the following. We assume that $\Omega$ is a cylinder:

$$
\Omega:=\left\{(x, y, z) \in \mathbb{R}^{3}: x^{2}+y^{2}<1,0<z<1\right\} .
$$

We consider a particular choice for functional $\mathcal{L}$ from (2.3), letting $\mathbf{g} \equiv \mathbf{0}$ and letting $\mathbf{f} \in$ $L^{2}\left(\Omega, \mathbb{R}^{3}\right)$ have a specific form. Namely, we let

$$
\mathcal{L}(\mathbf{u})=\int_{\Omega} \mathbf{f} \cdot \mathbf{u} \quad \text { with } \quad \mathbf{f}(x, y, z):=\left(\varphi_{x}(x, y), \varphi_{y}(x, y), \psi(z)\right),
$$


where $\varphi$ and $\psi$ satisfy the following restrictions (where $B$ denotes the unit ball centered at the origin in the $x y$ plane and $\Delta$ denotes the Laplacian in the $x, y$ variables):

$\varphi \in C^{2}(\bar{B})$ is radial, there exists $(x, y) \in B$ such that $\Delta \varphi(x, y) \neq 0$,

$\phi(1)=\phi^{\prime}(1)=\int_{0}^{1} r^{2} \phi^{\prime}(r) d r=0$, where $\phi$ denotes the radial profile of $\varphi$,

$$
\psi \in C^{0}([0,1]), \quad \int_{0}^{1} \psi(z) d z=0, \quad \int_{0}^{1} z \psi(z) d z \geq 0 .
$$

Condition (f 1) can be satisfied by choosing for instance $\varphi$ to be a suitable polynomial in the radial variable, like $\phi(r)=4 r^{6}-9 r^{4}+6 r^{2}-1$. As we will discuss in Sect. 5, with such choices of $\Omega$ and $\mathcal{L}$ the conditions $(\mathcal{L} 1)$ and $(\mathcal{L} 2)$ are satisfied and moreover $\{\mathbf{I}\} \subsetneq \mathcal{S}_{\mathcal{L}}^{0}$. We also have $\mathcal{S}_{\mathcal{L}}^{0} \subsetneq S O(3)$ if $\int_{0}^{1} z \psi(z) d z>0$ and $\mathcal{S}_{\mathcal{L}}^{0} \equiv S O(3)$ if $\int_{0}^{1} z \psi(z) d z=0$.

Concerning the strain energy functionals, we choose any function $\mathcal{W}(\mathbf{x}, \mathbf{F})=\mathcal{W}(\mathbf{F})$ satisfying assumptions $(\mathcal{W} \mathbf{1}),(\mathcal{W} 2),(\mathcal{W} 3),(\mathcal{W} 4)$ and being such that

$$
\frac{1}{2} \mathbf{F}^{T} D^{2} \mathcal{W}(\mathbf{I}) \mathbf{F}=4|\mathbf{F}|^{2} \quad \forall \mathbf{F} \in \mathbb{R}^{3 \times 3} .
$$

Accordingly we let $\mathcal{W}^{I}(\mathbf{x}, \mathbf{F})=\mathcal{W}^{I}(\mathbf{F})$ be equal to $\mathcal{W}(\mathbf{F})$ if $\operatorname{det} \mathbf{F}=1$ and equal to $+\infty$ otherwise. An example is the homogeneous Kirchoff - Saint-Venant energy, obtained by setting

$$
\mathcal{W}(\mathbf{x}, \mathbf{F})=\mathcal{W}(\mathbf{F})=\left|\mathbf{F}^{T} \mathbf{F}-\mathbf{I}\right|^{2} .
$$

We have the following

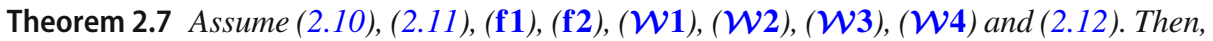
the assumptions of Theorem 2.4 and of Theorem 2.5 are satisfied, (2.8) holds true, and if $\|\psi\|_{L^{2}(0,1)}$ is small enough (2.9) holds true as well.

Remark 2.8 In the assumptions of Theorem 2.7, let us consider the rescaled displacement fields $\mathbf{v}_{j}$ and the generalized rescaled displacement fields $\mathbf{u}_{j}$ associated to a sequence of quasi-minimizers $\left(\mathbf{y}_{j}\right) \subset W^{1, p}\left(\Omega, \mathbb{R}^{3}\right)$ of $\mathcal{G}_{h_{j}}\left(\right.$ or $\left.\mathcal{G}_{h_{j}}^{I}\right)$. Since $\mathbf{v}_{j}(\mathbf{x})=h_{j}^{-1}\left(\mathbf{y}_{j}(\mathbf{x})-\mathbf{x}\right)$ and $\mathbf{u}_{j}(\mathbf{x})=h_{j}^{-1}\left(\mathbf{R}_{j}^{T} \mathbf{y}_{j}(\mathbf{x})-\mathbf{x}\right)$, where $\mathbf{R}_{j} \in \mathcal{A}_{p}\left(\mathbf{y}_{j}\right)$, we have

$$
\mathbb{E}\left(\mathbf{v}_{j}\right)=\frac{\mathbf{R}_{j}^{T}+\mathbf{R}_{j}-2 \mathbf{I}}{2 h_{j}}-\operatorname{sym}\left(\mathbf{R}_{j} \nabla \mathbf{u}_{j}\right) .
$$

Along a suitable subsequence we have $\nabla \mathbf{u}_{j} \rightarrow \nabla \mathbf{u}$ weakly in $L^{p}\left(\Omega, \mathbb{R}^{3 \times 3}\right)$ and $\mathbf{R}_{j} \rightarrow \mathbf{R}_{*} \in$ $\mathcal{S}_{\mathcal{L}}^{0}$ as we will show in Sect. 4. However, along the same sequence, $\mathbb{E}\left(\mathbf{v}_{j}\right)$ is unbounded in $L^{p}\left(\Omega, \mathbb{R}^{3 \times 3}\right)$, otherwise the results of [10] and [12] would entail convergence to the minimal value of the standard linearized elasticity functional, in contrast with Theorem 2.7. In fact, Theorem 2.7 shows that $\mathbf{R}_{*} \neq \mathbf{I}$ : the optimal rotation at a minimizer $\mathbf{u}$ of $\mathcal{G}\left(\right.$ or $\mathcal{G}^{I}$ ) is the limit of the rotations $\mathbf{R}_{j}$, it is not the identity matrix and then (2.13) confirms that $\mathbb{E}\left(\mathbf{v}_{j}\right)$ is unbounded in $L^{p}\left(\Omega, \mathbb{R}^{3 \times 3}\right)$.

\section{Rotated external forces with no gap}

Going back to the general setting, it is clear that a necessary condition for the validity of (2.8) and (2.9) is that $\mathcal{S}_{\mathcal{L}}^{0}$ is not reduced to the identity matrix. However, such a condition 
is not sufficient for the presence of a gap with linear elasticity. This is immediately seen by choosing $\mathbf{f} \equiv \mathbf{0}$ and $\mathbf{g} \equiv \mathbf{0}$, in which case $\mathcal{S}_{\mathcal{L}}^{0} \equiv S O(3)$ but of course all the four minima appearing in (2.8)-(2.9) are equal to zero. A much more general result holds, showing that the appearance of the gap is strongly influenced by the initial choice of the external loads. Before providing such result, we introduce some further notation. For every $\mathbf{R} \in S O$ (3), let

$$
\begin{aligned}
& \mathcal{G}_{h, \mathbf{R}}(\mathbf{y}):=h^{-2} \int_{\Omega} \mathcal{W}(\mathbf{x}, \nabla \mathbf{y}) d \mathbf{x}-h^{-1} \mathcal{L}_{\mathbf{R}}(\mathbf{y}(\mathbf{x})-\mathbf{x}), \quad \mathbf{y} \in W^{1, p}\left(\Omega, \mathbb{R}^{3}\right), \\
& \mathcal{G}_{\mathbf{R}}(\mathbf{v}):= \begin{cases}\int_{\Omega} \mathcal{Q}(\mathbf{x}, \mathbb{E}(\mathbf{v})) d \mathbf{x}-\max _{\hat{\mathbf{R}} \mathcal{S}_{\mathcal{L}_{\mathbf{R}}}} \mathcal{L}_{\mathbf{R}}(\hat{\mathbf{R}} \mathbf{v}) \text { if } \mathbf{v} \in H^{1}\left(\Omega, \mathbb{R}^{3}\right) \\
+\infty & \text { otherwise in } W^{1, p}\left(\Omega, \mathbb{R}^{3}\right),\end{cases}
\end{aligned}
$$

where $\mathcal{L}_{\mathbf{R}}: W^{1, p}\left(\Omega, \mathbb{R}^{3}\right) \rightarrow \mathbb{R}$ is defined as

$$
\mathcal{L}_{\mathbf{R}}(\mathbf{v}):=\mathcal{L}(\mathbf{R} \mathbf{v})=\int_{\Omega} \mathbf{R}^{T} \mathbf{f} \cdot \mathbf{v} d \mathbf{x}-\int_{\partial \Omega} \mathbf{R}^{T} \mathbf{g} \cdot \mathbf{v} d \mathcal{H}^{2}(\mathbf{x}),
$$

so that $\mathcal{L}_{\mathbf{R}}$ is just the usual load functional associated to the external forces $\mathbf{R}^{T} \mathbf{f}, \mathbf{R}^{T} \mathbf{g}$. Similarly, $\mathcal{E}_{\mathbf{R}}$ is defined by replacing $\mathcal{L}$ with $\mathcal{L}_{\mathbf{R}}$ in the definition of $\mathcal{E}$. The corresponding functionals $\mathcal{G}_{h, \mathbf{R}}^{I}, \mathcal{G}_{\mathbf{R}}^{I}, \mathcal{E}_{\mathbf{R}}^{I}$ of incompressible elasticity are also defined by replacing $\mathcal{L}$ with $\mathcal{L}_{\mathbf{R}}$ in the definition of $\mathcal{G}_{h}^{I}, \mathcal{G}^{I}$ and $\mathcal{E}^{I}$, respectively.

Remark 2.9 Since $\mathcal{L}$ satisfies $(\mathcal{L} 1)$ and $(\mathcal{L} 2)$, it is clear that given $\mathbf{R} \in \mathcal{S}_{\mathcal{L}}^{0}$ there holds $\mathcal{L}_{\mathbf{R}}(\mathbf{c})=0$ for every $\mathbf{c} \in \mathbb{R}^{3}$ and

$$
\mathcal{L}_{\mathbf{R}}((\mathbf{S}-\mathbf{I}) \mathbf{x})=\mathcal{L}((\mathbf{R S}-\mathbf{I}) \mathbf{x})-\mathcal{L}((\mathbf{R}-\mathbf{I}) \mathbf{x})=\mathcal{L}((\mathbf{R S}-\mathbf{I}) \mathbf{x}) \leq 0
$$

for every $\mathbf{S} \in S O(3)$, thus showing that $\mathcal{L}_{\mathbf{R}}$ satisfies $(\mathcal{L} 1)$ and $(\mathcal{L} 2)$ as well. Therefore, by Theorem 2.4 and Theorem 2.5, functionals $\mathcal{G}_{\mathbf{R}}$ and $\mathcal{G}_{\mathbf{R}}^{I}$ can be viewed as the limit of functionals $\mathcal{G}_{h, \mathbf{R}}$ and $\mathcal{G}_{h, \mathbf{R}}^{I}$ respectively.

Theorem 2.10 Assume $(\mathcal{L} 1),(\mathcal{L} 2),(\mathcal{W} 1),(\mathcal{W} 2),(\mathcal{W} 3),(\mathcal{W} 4)$. If $\mathbf{R} \in \mathcal{S}_{\mathcal{L}}^{0}$, the rotated load functional $\mathcal{L}_{\mathbf{R}}$ still satisfies $(\mathcal{L} 1)$ and $(\mathcal{L} 2)$, and $\mathcal{S}_{\mathcal{L}}^{0} \equiv \mathcal{S}_{\mathcal{L}_{\mathbf{R}}}^{0}$. Moreover, if $\mathbf{u}$ minimizes $\mathcal{G}$ (resp. $\mathcal{G}^{I}$ ) over $W^{1, p}\left(\Omega, \mathbb{R}^{3}\right)$ and $\mathbf{R} \in \mathcal{S}_{\mathcal{L}}^{0}$ realizes the maximum in the definition of $\mathcal{G}(\mathbf{u})$ (resp. $\mathcal{G}^{I}(\mathbf{u})$ ), then $\mathbf{u}$ minimizes $\mathcal{G}_{\mathbf{R}}$ (resp. $\left.\mathcal{G}_{\mathbf{R}}^{I}\right)$ over $W^{1, p}\left(\Omega, \mathbb{R}^{3}\right)$ and

$$
\min _{W^{1, p}\left(\Omega, \mathbb{R}^{3}\right)} \mathcal{G}_{\mathbf{R}}=\min _{W^{1, p}\left(\Omega, \mathbb{R}^{3}\right)} \mathcal{E}_{\mathbf{R}} \quad\left(\text { resp. } \min _{W^{1, p}\left(\Omega, \mathbb{R}^{3}\right)} \mathcal{G}_{\mathbf{R}}^{I}=\min _{W^{1, p}\left(\Omega, \mathbb{R}^{3}\right)} \mathcal{E}_{\mathbf{R}}^{I}\right) .
$$

Remark 2.11 Given any external forces $\mathbf{f}$, $\mathbf{g}$ satisfying $(\mathcal{L} 1)-(\mathcal{L} 2)$, the above theorem yields the existence of new external forces satisfying $(\mathcal{L} 1)-(\mathcal{L} 2)$, having the same rotation kernel as $\mathbf{f}, \mathbf{g}$, for which there is no gap with linear elasticity.

We include in this section the straightforward proof of Theorem 2.10.

Proof of Theorem 2.10. We give the proof for the compressible case, the arguments for the incompressible case being the very same.

By Remark 2.11, $\mathcal{L}_{\mathbf{R}}$ satisfies $(\mathcal{L} 1)$ and $(\mathcal{L} 2)$. If $\mathbf{R} \in \mathcal{S}_{\mathcal{L}}^{0}$ we notice that, since $\mathcal{S}_{\mathcal{L}}^{0}$ and $\mathcal{S}_{\mathcal{L}_{\mathbf{R}}}^{0}$ are subgroups of $S O(3)$ as shown in Remark 2.2, inequality (2.15) is an equality as soon as $\mathbf{S} \in \mathcal{S}_{\mathcal{L}}^{0}$, so that $\mathcal{S}_{\mathcal{L}_{\mathbf{R}}}^{0} \supseteq \mathcal{S}_{\mathcal{L}}^{0}$. Still assuming $\mathbf{R} \in \mathcal{S}_{\mathcal{L}}^{0}$, we may also prove the opposite 
inclusion: indeed, by the inclusion $\mathcal{S}_{\mathcal{L}_{\mathbf{R}}}^{0} \supseteq \mathcal{S}_{\mathcal{L}}^{0}$ we deduce that $\mathbf{R} \in \mathcal{S}_{\mathcal{L}_{\mathbf{R}}}^{0}$, so that again Remark 2.2 implies $\mathbf{R}^{T} \in \mathcal{S}_{\mathcal{L}_{\mathbf{R}}}^{0}$ and $\mathbf{R}^{T} \mathbf{S} \in \mathcal{S}_{\mathcal{L}_{\mathbf{R}}}^{0}$ for every $\mathbf{S} \in \mathcal{S}_{\mathcal{L}_{\mathbf{R}}}^{0}$, thus

$$
\mathcal{L}((\mathbf{S}-\mathbf{I}) \mathbf{x})=\mathcal{L}_{\mathbf{R}}\left(\mathbf{R}^{T}(\mathbf{S}-\mathbf{I}) \mathbf{x}\right)=\mathcal{L}_{\mathbf{R}}\left(\left(\mathbf{R}^{T} \mathbf{S}-\mathbf{I}\right) \mathbf{x}\right)-\mathcal{L}_{\mathbf{R}}\left(\left(\mathbf{R}^{T}-\mathbf{I}\right) \mathbf{x}\right)=0
$$

for every $\mathbf{S} \in \mathcal{S}_{\mathcal{L}_{\mathbf{R}}}^{0}$, proving that $\mathcal{S}_{\mathcal{L}_{\mathbf{R}}}^{0} \subseteq \mathcal{S}_{\mathcal{L}}^{0}$.

Let now $\mathbf{R} \in \mathcal{S}_{\mathcal{L}}^{0}$ realize the maximum in the definition of $\mathcal{G}(\mathbf{u})$, where $\mathbf{u}$ minimizes $\mathcal{G}$ over $W^{1, p}\left(\Omega, \mathbb{R}^{3}\right)$. We conclude by checking that $\mathbf{u}$ is also a minimizer of $\mathcal{G}_{\mathbf{R}}$ over $W^{1, p}\left(\Omega, \mathbb{R}^{3}\right)$ and that the identity matrix realizes the maximum in the definition of $\mathcal{G}_{\mathbf{R}}(\mathbf{u})$. We proceed by contradiction, supposing that there are $\tilde{\mathbf{u}} \in W^{1, p}\left(\Omega, \mathbb{R}^{3}\right)$ and $\tilde{\mathbf{R}} \in \mathcal{S}_{\mathcal{L}_{\mathbf{R}}}^{0}$ such that

$$
\min _{W^{1, p}\left(\Omega, \mathbb{R}^{3}\right)} \mathcal{G}_{\mathbf{R}}=\int_{\Omega} \mathcal{Q}(\mathbf{x}, \mathbb{E}(\tilde{\mathbf{u}})) d \mathbf{x}-\mathcal{L}_{\mathbf{R}}(\tilde{\mathbf{R}} \tilde{\mathbf{u}})<\int_{\Omega} \mathcal{Q}(\mathbf{x}, \mathbb{E}(\mathbf{u})) d \mathbf{x}-\mathcal{L}_{\mathbf{R}}(\mathbf{u}) .
$$

Then, having shown that $\mathbf{R} \tilde{\mathbf{R}} \in \mathcal{S}_{\mathcal{L}}^{0}$, we deduce

$$
\begin{aligned}
\mathcal{G}(\tilde{\mathbf{u}}) & =\int_{\Omega} \mathcal{Q}(\mathbf{x}, \mathbb{E}(\tilde{\mathbf{u}})) d \mathbf{x}-\max _{\mathbf{S} \in \mathcal{S}_{\mathcal{L}}^{0}} \mathcal{L}(\mathbf{S} \tilde{\mathbf{u}}) \leq \int_{\Omega} \mathcal{Q}(\mathbf{x}, \mathbb{E}(\tilde{\mathbf{u}})) d \mathbf{x}-\mathcal{L}(\mathbf{R} \tilde{\mathbf{R}} \tilde{\mathbf{u}}) \\
& =\int_{\Omega} \mathcal{Q}(\mathbf{x}, \mathbb{E}(\tilde{\mathbf{u}})) d \mathbf{x}-\mathcal{L}_{\mathbf{R}}(\tilde{\mathbf{R}} \tilde{\mathbf{u}})<\int_{\Omega} \mathcal{Q}(\mathbf{x}, \mathbb{E}(\mathbf{u})) d \mathbf{x}-\mathcal{L}_{\mathbf{R}}(\mathbf{u}) \\
& =\int_{\Omega} \mathcal{Q}(\mathbf{x}, \mathbb{E}(\mathbf{u})) d \mathbf{x}-\mathcal{L}(\mathbf{R u})=\mathcal{G}(\mathbf{u})
\end{aligned}
$$

which is a contradiction with the minimality of $\mathbf{u}$ for $\mathcal{G}$.

Remark 2.12 We close this section by mentioning a difference between our approach to Theorem 2.4 and the one in [13], where the authors introduce the set $\mathcal{R}:=\operatorname{argmax}_{\mathbf{R} \in S O \text { (3) }} \mathcal{L}(\mathbf{R x})$. Under the usual assumptions on $\mathcal{W}$ (with $p=2$ ) and assuming only $(\mathcal{L} \mathbf{1}$ ), it follows from [13, Theorem 5.3] that for every $\mathbf{U} \in \mathcal{R}$ the infimum of

$$
\mathcal{J}_{h, \mathbf{U}}(\mathbf{y}):=h^{-2} \int_{\Omega} \mathcal{W}(\mathbf{x}, \nabla \mathbf{y}) d \mathbf{x}-h^{-1} \mathcal{L}(\mathbf{y}-\mathbf{U x})
$$

among all $\mathbf{u} \in H^{1}\left(\Omega, \mathbb{R}^{3}\right)$ converges as $h \rightarrow 0$ to the minimum over $\mathbf{u} \in H^{1}\left(\Omega, \mathbb{R}^{3}\right)$ of

$$
\mathcal{J}_{\mathbf{U}}(\mathbf{u})=\int_{\Omega} \mathcal{Q}(\mathbf{x}, \mathbb{E}(\mathbf{u})) d \mathbf{x}-\max _{\mathbf{R} \in \mathcal{R}} \mathcal{L}(\mathbf{U R u}) .
$$

It is readily seen that if $\mathcal{L}$ satisfies $(\mathcal{L} 1)$ and $\mathbf{I} \in \mathcal{R}$ then $\mathcal{L}$ satisfies $(\mathcal{L} 2), \mathcal{R} \equiv \mathcal{S}_{\mathcal{L}}^{0}$ and $\mathcal{J}_{\mathbf{I}}(\mathbf{u})=\mathcal{G}(\mathbf{u})$ for every $\mathbf{u} \in H^{1}\left(\Omega, \mathbb{R}^{3}\right)$. Moreover

$$
\mathcal{J}_{h, \mathbf{U}}(\mathbf{y})=\mathcal{G}_{h}(\mathbf{y})+h^{-1} \mathcal{L}(\mathbf{U} \mathbf{x}-\mathbf{x}) \geq \mathcal{G}_{h}(\mathbf{y}),
$$

and equality holds if and only if $\mathbf{I} \in \mathcal{R}$ so that in this case $\inf \mathcal{G}_{h} \rightarrow \min \mathcal{G}=\min \mathcal{J}_{\mathbf{I}}$. Therefore the results of [13] imply Theorem (2.4) when $p=2$. On the other hand, if $\mathbf{I} \notin \mathcal{R}$ (so that $(\mathcal{L} 2)$ does not hold) then $\inf \mathcal{G}_{h} \rightarrow-\infty$ as $h \rightarrow 0$ (see Remark 2.6) and therefore hypothesis $(\mathcal{L} 2)$ cannot be dropped in Theorem (2.4).

In addition, we claim that whenever the sole condition $(\mathcal{L} \mathbf{1})$ holds along with the usual assumptions on $\mathcal{W}$ with $p \in(1,2]$, Theorem (2.4) implies that for every $\mathbf{U} \in \mathcal{R}$ there holds inf $\mathcal{J}_{h, \mathbf{U}} \rightarrow \min \mathcal{J}$ as $h \rightarrow 0$, where

$$
\mathcal{J}(\mathbf{u})=\int_{\Omega} \mathcal{Q}(\mathbf{x}, \mathbb{E}(\mathbf{u})) d \mathbf{x}-\max _{\mathbf{R} \in \mathcal{R}} \mathcal{L}(\mathbf{R u}) .
$$


Indeed, we first notice that if $\mathcal{L}$ satisfies $(\mathcal{L} \mathbf{1})$, then $\mathbf{U} \in \mathcal{R}$ implies that $\mathcal{L}_{\mathbf{U}}$ satisfies both $(\mathcal{L} \mathbf{1})$ and $(\mathcal{L} 2)$ and that

$$
\mathcal{R}=\left\{\mathbf{U R}: \mathbf{R} \in \mathcal{S}_{\mathcal{L}_{\mathbf{U}}}^{0}\right\}
$$

In fact, given $\mathbf{U} \in \mathcal{R}$, if $\mathbf{S} \in \mathcal{S}_{\mathcal{L}_{\mathbf{U}}}^{0}$ it is immediately seen that $\mathbf{U S} \in \mathcal{R}$, and given any $\mathbf{S}_{*} \in \mathcal{R}$ we may write $\mathbf{S}_{*}=\mathbf{U} \mathbf{U}^{T} \mathbf{S}_{*}$ and it is immediately seen that $\mathbf{U}^{T} \mathbf{S}_{*} \in \mathcal{S}_{\mathcal{L}_{\mathbf{U}}}^{0}$, thus proving (2.17). Moreover we notice that

$$
\mathcal{J}_{h, \mathbf{U}}(\mathbf{y})=\mathcal{G}_{h, \mathbf{U}}\left(\mathbf{U}^{T} \mathbf{y}\right)=h^{-2} \int_{\Omega} \mathcal{W}\left(\mathbf{x}, \nabla\left(\mathbf{U}^{T} \mathbf{y}\right)\right) d \mathbf{x}-h^{-1} \mathcal{L}_{\mathbf{U}}\left(\mathbf{U}^{T} \mathbf{y}-\mathbf{x}\right),
$$

thus inf $\mathcal{J}_{h, \mathbf{U}}=\inf \mathcal{G}_{h, \mathbf{U}}$, and by recalling that $\mathcal{L}_{\mathbf{U}}$ satisfies both $(\mathcal{L} 1)$ and $(\mathcal{L} 2)$, it follows from Theorem 2.4 that inf $\mathcal{J}_{h, \mathbf{U}}$ converges, as $h \rightarrow 0$, to the minimum of

$$
\mathcal{G}_{\mathbf{U}}(\mathbf{u}):=\int_{\Omega} \mathcal{Q}(\mathbf{x}, \mathbb{E}(\mathbf{u})) d \mathbf{x}-\max _{\mathbf{R} \in \mathcal{S}_{\mathcal{L}_{\mathbf{U}}}^{0}} \mathcal{L}_{\mathbf{U}}(\mathbf{R u})
$$

among all $\mathbf{u} \in H^{1}\left(\Omega, \mathbb{R}^{3}\right)$. By recalling (2.14) and by exploiting (2.17) we also get

$$
\max _{\mathbf{R} \in \mathcal{S}_{\mathcal{L}_{\mathbf{U}}}^{0}} \mathcal{L}_{\mathbf{U}}(\mathbf{R u})=\max _{\mathbf{R} \in \mathcal{S}_{\mathcal{L}_{\mathbf{U}}}^{0}} \mathcal{L}(\mathbf{U R \mathbf { R }})=\max _{\mathbf{R} \in \mathcal{R}} \mathcal{L}(\mathbf{R u})
$$

that is, $\mathcal{G}_{\mathbf{U}}(\mathbf{u}) \equiv \mathcal{J}(\mathbf{u})$ and $\inf \mathcal{J}_{h, \mathbf{U}} \rightarrow \min \mathcal{J}$ thus proving the claim (i.e., $\min \mathcal{J}=\min \mathcal{J}_{\mathbf{U}}$ for every $\mathbf{U} \in \mathcal{R})$.

In any case, we observe that if $\Omega$ is the reference configuration of the elastic body, the second term on the right hand side of (2.16) represents the work expended by the given external forces $\mathbf{f}, \mathbf{g}$ if and only $\mathbf{I} \in \mathcal{R}$ or equivalently if and only if $(\mathcal{L} 2)$ is satisfied by $\mathbf{f}, \mathbf{g}$.

\section{Preliminary results}

\section{Some properties of $\mathcal{W}$}

The frame indifference assumption $(\mathcal{W} \mathbf{1})$ implies that there exists a function $\mathcal{V}$ such that for a.e. $\mathbf{x} \in \Omega$

$$
\mathcal{W}(\mathbf{x}, \mathbf{F})=\mathcal{V}\left(\mathbf{x}, \frac{1}{2}\left(\mathbf{F}^{T} \mathbf{F}-\mathbf{I}\right)\right) \quad \forall \mathbf{F} \in \mathbb{R}^{3 \times 3} .
$$

By $(\mathcal{W} 3)$, for a.e. $\mathbf{x} \in \Omega$, we have $\mathcal{W}(\mathbf{x}, \mathbf{R})=D \mathcal{W}(\mathbf{x}, \mathbf{R})=0$ for any $\mathbf{R} \in S O$ (3). By (3.1), for a.e. $\mathbf{x} \in \Omega$, given $\mathbf{B} \in \mathbb{R}^{3 \times 3}$ and $h>0$ we have $\mathcal{W}(\mathbf{x}, \mathbf{I}+h \mathbf{B})=\mathcal{V}\left(\mathbf{x}, h \operatorname{sym} \mathbf{B}+\frac{1}{2} h^{2} \mathbf{B}^{T} \mathbf{B}\right)$ and $(\mathcal{W} 3)$ again implies

$\lim _{h \rightarrow 0} h^{-2} \mathcal{W}(\mathbf{x}, \mathbf{I}+h \mathbf{B})=\frac{1}{2} \operatorname{sym} \mathbf{B} D^{2} \mathcal{V}(\mathbf{x}, \mathbf{0}) \operatorname{sym} \mathbf{B}=\frac{1}{2} \mathbf{B}^{T} D^{2} \mathcal{W}(\mathbf{x}, \mathbf{I}) \mathbf{B}, \quad \forall \mathbf{B} \in \mathbb{R}^{3 \times 3}$.

By the latter and by $(\mathcal{W} 4)$, for a.e. $\mathbf{x} \in \Omega$, the following holds for every $\mathbf{B} \in \mathbb{R}^{3 \times 3}$ with $\operatorname{det} \mathbf{B}>0$ :

$$
\begin{aligned}
\frac{1}{2} \mathbf{B}^{T} D^{2} \mathcal{W}(\mathbf{x}, \mathbf{I}) \mathbf{B} & =\lim _{h \rightarrow 0} h^{-2} \mathcal{W}(\mathbf{x}, \mathbf{I}+h \mathbf{B}) \geq \limsup _{h \rightarrow 0} C h^{-2} d^{2}(\mathbf{I}+h \mathbf{B}, S O(3)) \\
& =\limsup _{h \rightarrow 0} C h^{-2}\left|\sqrt{(\mathbf{I}+h \mathbf{B})^{T}(\mathbf{I}+h \mathbf{B})}-\mathbf{I}\right|^{2}=C|\operatorname{sym} \mathbf{B}|^{2} .
\end{aligned}
$$


Moreover, as noticed also in [12], by expressing the remainder of Taylor's expansion in terms of the $\mathbf{x}$-independent modulus of continuity $\omega$ of $D^{2} \mathcal{W}(\mathbf{x}, \cdot)$ on the set $\mathcal{U}$ from $(\mathcal{W} 3)$, we have

$$
\left|\mathcal{W}(\mathbf{x}, \mathbf{I}+h \mathbf{B})-\frac{h^{2}}{2} \operatorname{sym} \mathbf{B} D^{2} \mathcal{W}(\mathbf{x}, \mathbf{I}) \operatorname{sym} \mathbf{B}\right| \leq h^{2} \omega(h|\mathbf{B}|)|\mathbf{B}|^{2}
$$

for any small enough $h$ (such that $h \mathbf{B} \in \mathcal{U}$ ). Similarly, $\mathcal{V}(\mathbf{x}, \cdot)$ is $C^{2}$ in a neighborhood of the origin in $\mathbb{R}^{3 \times 3}$, with an $\mathbf{x}$-independent modulus of continuity $\eta: \mathbb{R}_{+} \rightarrow \mathbb{R}$, which is increasing and such that $\lim _{t \rightarrow 0^{+}} \eta(t)=0$, and we have

$$
\left|\mathcal{V}(\mathbf{x}, h \mathbf{B})-\frac{h^{2}}{2} \operatorname{sym} \mathbf{B} D^{2} \mathcal{V}(\mathbf{x}, \mathbf{0}) \operatorname{sym} \mathbf{B}\right| \leq h^{2} \eta(h|\mathbf{B}|)|\mathbf{B}|^{2}
$$

for any small enough $h$.

\section{Some functional inequalities}

Let $p \in(1,2]$. Since $\Omega$ is a bounded open connected Lipschitz set, by Sobolev embedding, Sobolev trace embedding and by the Poincaré inequality for any $\mathbf{v} \in W^{1, p}\left(\Omega, \mathbb{R}^{3}\right)$ there exists $\overline{\mathbf{c}}, \overline{\mathbf{d}} \in \mathbb{R}^{3}$ such that

$$
\|\mathbf{v}-\overline{\mathbf{c}}\|_{L^{\frac{3 p}{3-p}}\left(\Omega, \mathbb{R}^{3}\right)}+\|\mathbf{v}-\overline{\mathbf{d}}\|_{L^{\frac{2 p}{3-p}}\left(\partial \Omega, \mathbb{R}^{3}\right)} \leq K\|\nabla \mathbf{v}\|_{L^{p}\left(\Omega, \mathbb{R}^{3 \times 3}\right)},
$$

where $K$ is a constant only depending on $\Omega, p$. Moreover, the second Korn inequality (see for instance [14]), combined with Sobolev and trace inequalities, provides the existence of a further constant $C_{K}=C_{K}(\Omega, p)$ such that for all $\mathbf{v} \in W^{1, p}\left(\Omega, \mathbb{R}^{3}\right)$

$$
\|\mathbf{v}-\mathbb{P} \mathbf{v}\|_{L^{\frac{3 p}{3-p}}\left(\Omega, \mathbb{R}^{3}\right)}+\|\mathbf{v}-\mathbb{P} \mathbf{v}\|_{L^{\frac{2 p}{3-p}}\left(\partial \Omega, \mathbb{R}^{3}\right)} \leq C_{K}\|\mathbb{E}(\mathbf{v})\|_{L^{p}\left(\Omega, \mathbb{R}^{3 \times 3}\right)},
$$

where $\mathbb{P}$ denotes the projection operator on infinitesimal rigid displacements, i.e., on the set of displacement fields $\mathbf{v}$ such that $\mathbb{E}(\mathbf{v})=0$.

A useful consequence of (3.4), if $(\mathcal{L} \mathbf{1})$ holds true, is the following estimate. Since for any $\mathbf{v} \in W^{1, p}\left(\Omega, \mathbb{R}^{3}\right)$ and for every $\mathbf{c}, \mathbf{d} \in \mathbb{R}^{3}$

$$
|\mathcal{L}(\mathbf{v})| \leq\|\mathbf{f}\|_{L^{\frac{3 p}{4 p-3}}\left(\Omega, \mathbb{R}^{3}\right)}\|\mathbf{v}-\mathbf{c}\|_{L^{\frac{3 p}{3-p}}\left(\Omega, \mathbb{R}^{3}\right)}+\|\mathbf{g}\|_{L^{\frac{2 p}{3 p-3}}\left(\partial \Omega, \mathbb{R}^{3}\right)}\|\mathbf{v}-\mathbf{d}\|{ }_{L^{\frac{2 p}{3-p}}\left(\partial \Omega, \mathbb{R}^{3}\right)}
$$

then (3.4) implies

$$
|\mathcal{L}(\mathbf{v})| \leq C_{\mathcal{L}}\|\nabla \mathbf{v}\|_{L^{p}\left(\Omega, \mathbb{R}^{3 \times 3}\right)}
$$

where $C_{\mathcal{L}}:=K\left(\|\mathbf{f}\|_{L^{\frac{3 p}{4 p-3}}\left(\Omega, \mathbb{R}^{3}\right)}+\|\mathbf{g}\|_{L^{\frac{2 p}{3 p-3}}\left(\partial \Omega, \mathbb{R}^{3}\right)}\right)$ and $K$ is the constant in (3.4). By Young inequality we then obtain

$$
|\mathcal{L}(\mathbf{v})| \leq \frac{p-1}{p}\left(C_{\mathcal{L}^{\varepsilon}} \varepsilon^{-1}\right)^{\frac{p}{p-1}}+p^{-1} \varepsilon^{p}\|\nabla \mathbf{v}\|_{L^{p}\left(\Omega, \mathbb{R}^{3 \times 3}\right)}^{p}
$$

for every $\varepsilon>0$. 


\section{Convergence of minimizers: Proof of Theorem 2.4 and Theorem 2.5}

\subsection{The incompressible case}

We give the proof of our convergence result regarding the incompressible case, which is the more difficult. We will briefly show how to adapt the arguments to the compressible case later on. The proof follows the standard line of a $\Gamma$-convergence argument: we prove compactness, a lower bound and an upper bound.

Lemma 4.1 (Compactness). Assume $(\mathcal{W} 1),(\mathcal{W} 2),(\mathcal{W} 3),(\mathcal{W} 4),(\mathcal{L} 1)$ and $(\mathcal{L} 2)$. Let $\left(h_{j}\right)_{j \subset \mathbb{N}} \subset$ $(0,1)$ be a vanishing sequence, let $M>0$ and let $\left(\mathbf{y}_{j}\right)_{j \in \mathbb{N}} \subset W^{1, p}\left(\Omega, \mathbb{R}^{3}\right)$ be a sequence such that

$$
\mathcal{G}_{h_{j}}^{I}\left(\mathbf{y}_{j}\right) \leq M \quad \forall j \in \mathbb{N} .
$$

Let $\mathbf{R}_{j} \in \mathcal{A}_{p}\left(\mathbf{y}_{j}\right)$ and $\mathbf{u}_{j}(\mathbf{x}):=h_{j}^{-1}\left(\mathbf{R}_{j}^{T} \mathbf{y}_{j}(\mathbf{x})-\mathbf{x}\right)$. Then, the sequence $\left(\nabla \mathbf{u}_{j}\right)_{j \in \mathbb{N}}$ is bounded in $L^{p}\left(\Omega, \mathbb{R}^{3 \times 3}\right)$ and any of its weak $L^{p}\left(\Omega, \mathbb{R}^{3 \times 3}\right)$ limit points is of the form $\nabla \mathbf{u}_{*}$ for some $\mathbf{u}_{*} \in H^{1}\left(\Omega, \mathbb{R}^{3}\right)$. Moreover, any limit point of the sequence $\left(\mathbf{R}_{j}\right)_{j \in \mathbb{N}} \subset S O$ (3) belongs to $\mathcal{S}_{\mathcal{L}}^{0}$.

Proof By (4.1) we obtain det $\nabla \mathbf{y}_{j}=1$ for any $j \in \mathbb{N}$. By (4.1), $(\mathcal{L} 1),(\mathcal{L} 2)$ and (3.6) we get for every $\varepsilon>0$

$$
\begin{aligned}
h_{j}^{-2} \int_{\Omega} \mathcal{W}^{I}\left(\mathbf{x}, \nabla \mathbf{y}_{j}\right) d \mathbf{x} & =h_{j}^{-2} \int_{\Omega} \mathcal{W}^{I}\left(\mathbf{x}, \nabla \mathbf{y}_{j}\right) d \mathbf{x} \leq M+h_{j}^{-1} \mathcal{L}\left(\mathbf{y}_{j}-\mathbf{x}\right) \\
& =M+h_{j}^{-1} \mathcal{L}\left(\mathbf{y}_{j}-\mathbf{R}_{j} \mathbf{x}\right)+h_{j}^{-1} \mathcal{L}\left(\left(\mathbf{R}_{j}-\mathbf{I}\right) \mathbf{x}\right) \\
& \leq M+h_{j}^{-1} \mathcal{L}\left(\mathbf{y}_{j}-\mathbf{R}_{j} \mathbf{x}\right) \\
& \leq \frac{p-1}{p}\left(C_{\mathcal{L}} \varepsilon^{-1}\right)^{\frac{p}{p-1}}+p^{-1} \varepsilon^{p}\left\|\mathbf{R}_{j} \nabla \mathbf{u}_{j}\right\|_{L^{p}\left(\Omega, \mathbb{R}^{3 \times 3}\right)}^{p}
\end{aligned}
$$

Moreover, by recalling $(\mathcal{W} 4),(2.5)$ and (2.2), there exists a constant $C$ (only depending on $p$ and $\Omega$ ) such that for every $\varepsilon>0$

$$
\begin{gathered}
h_{j}^{-2} \int_{\Omega} \mathcal{W}^{I}\left(\mathbf{x}, \nabla \mathbf{y}_{j}\right) d \mathbf{x}=h_{j}^{-2} \int_{\Omega} \mathcal{W}^{I}\left(\mathbf{x}, \nabla \mathbf{y}_{j}\right) d \mathbf{x} \geq C h_{j}^{-2} \int_{\Omega} g_{p}\left(\left|\nabla \mathbf{y}_{j}-\mathbf{R}_{j}\right|\right) d \mathbf{x} \\
\quad=C h_{j}^{-2} \int_{\Omega} g_{p}\left(h_{j}\left|\mathbf{R}_{j} \nabla \mathbf{y}_{j}\right|\right) d \mathbf{x} \geq C\left\|\mathbf{R}_{j} \nabla \mathbf{u}_{j}\right\|_{L^{p}\left(\Omega, \mathbb{R}^{3 \times 3}\right)}^{p}-\frac{2-p}{p} C|\Omega|,
\end{gathered}
$$

which in combination with (4.2) entails, by taking small enough $\varepsilon$,

$$
\left\|\mathbf{R}_{j} \nabla \mathbf{u}_{j}\right\|_{L^{p}\left(\Omega, \mathbb{R}^{3 \times 3}\right)}^{p}=\left\|\nabla \mathbf{u}_{j}\right\|_{L^{p}\left(\Omega, \mathbb{R}^{3 \times 3}\right)}^{p} \leq Q
$$

for some suitable constant $Q$ depending only on $C_{\mathcal{L}}, p, \Omega$ (and not on $j$ ). On the other hand

$$
\mathcal{G}_{h}^{I}\left(\mathbf{y}_{j}\right):=h_{j}^{-2} \int_{\Omega} \mathcal{W}^{I}\left(\mathbf{x}, \nabla \mathbf{y}_{j}\right) d \mathbf{x}-\mathcal{L}\left(\mathbf{R}_{j} \mathbf{u}_{j}\right)-h_{j}^{-1} \mathcal{L}\left(\left(\mathbf{R}_{j}-\mathbf{I}\right) \mathbf{x}\right) \leq M
$$

entails

$$
0 \leq-h_{j}^{-1} \mathcal{L}\left(\left(\mathbf{R}_{j}-\mathbf{I}\right) \mathbf{x}\right) \leq M+\mathcal{L}\left(\mathbf{R}_{j} \mathbf{u}_{j}\right)
$$

and by (3.6), (4.4) we get $\mathcal{L}\left(\left(\mathbf{R}_{j}-\mathbf{I}\right) \mathbf{x}\right) \rightarrow 0$ as $j \rightarrow+\infty$. Therefore, if $\mathbf{R}_{j} \rightarrow \mathbf{R}_{*}$ along a suitable subsequence, we have $\mathcal{L}\left(\left(\mathbf{R}_{*}-\mathbf{I}\right) \mathbf{x}\right)=0$ that is $\mathbf{R}_{*} \in \mathcal{S}_{\mathcal{L}}^{0}$.

By (4.4), the sequence $\left(\nabla \mathbf{u}_{j}\right)_{j \in \mathbb{N}}$ is bounded in $L^{p}\left(\Omega, \mathbb{R}^{3 \times 3}\right)$. As a consequence of the Poincaré inequality, any of its weak $L^{p}\left(\Omega, \mathbb{R}^{3 \times 3}\right)$ limit points is of the form $\nabla \mathbf{u}_{*}$ for some 
$\mathbf{u}_{*} \in W^{1, p}\left(\Omega, \mathbb{R}^{3}\right)$. Assuming that $\nabla \mathbf{u}_{*}$ is the weak $L^{p}\left(\Omega, \mathbb{R}^{3 \times 3}\right)$ limit point along a not relabeled subsequence, we are only left to prove that $\mathbf{u}_{*} \in H^{1}\left(\Omega ; \mathbb{R}^{3}\right)$. To this aim we let

$$
B_{j}:=\left\{\mathbf{x} \in \Omega: \sqrt{h}_{j}\left|\nabla \mathbf{u}_{j}\right| \leq 1\right\}
$$

so that

$$
\int_{B_{j}}\left|\nabla \mathbf{u}_{j}\right|^{2} d \mathbf{x} \leq h_{j}^{-2} \int_{\Omega} g_{p}\left(h_{j}\left|\nabla \mathbf{u}_{j}\right|\right) d \mathbf{x}=h_{j}^{-2} \int_{\Omega} g_{p}\left(\left|\nabla \mathbf{y}_{j}-\mathbf{R}_{j}\right|\right) d \mathbf{x},
$$

hence by (4.2), (4.3) and (4.4) we get uniform boundedness in $L^{2}\left(\Omega, \mathbb{R}^{3 \times 3}\right)$ for the sequence $\left(\mathbf{1}_{B_{j}} \nabla \mathbf{u}_{j}\right)_{j \in \mathbb{N}}$ thus up to subsequences $\mathbf{1}_{B_{j}} \nabla \mathbf{u}_{j} \rightarrow \mathbf{w}$ weakly in $L^{2}\left(\Omega, \mathbb{R}^{3 \times 3}\right)$ as $j \rightarrow+\infty$. On the other hand for every $q \in(1, p)$ we have

$$
\int_{B_{j}^{c}}\left|\nabla \mathbf{u}_{j}\right|^{q} d \mathbf{x} \leq\left(\int_{B_{j}^{c}}\left|\nabla \mathbf{u}_{j}\right|^{p} d \mathbf{x}\right)^{q / p}\left|B_{j}^{c}\right|^{(p-q) / p}
$$

where the right hand side vanishes as $j \rightarrow+\infty$ since $\left|B_{j}^{c}\right| \rightarrow 0$ by Chebyshev inequality. By taking into account that

$$
\nabla \mathbf{u}_{j}=\mathbf{1}_{B_{j}^{c}} \nabla \mathbf{u}_{j}+\mathbf{1}_{B_{j}} \nabla \mathbf{u}_{j}
$$

and that $\mathbf{1}_{B_{j}} \nabla \mathbf{\mathbf { u } _ { j }} \rightarrow \mathbf{w}$ weakly in $L^{2}\left(\Omega, \mathbb{R}^{3 \times 3}\right)$ we get $\nabla \mathbf{u}_{j} \rightarrow \mathbf{w}$ weakly in $L^{q}\left(\Omega, \mathbb{R}^{3 \times 3}\right)$ and recalling that $\nabla \mathbf{u}_{j} \rightarrow \nabla \mathbf{u}_{*}$ weakly in $L^{p}\left(\Omega, \mathbb{R}^{3 \times 3}\right)$ we get $\mathbf{w}=\nabla \mathbf{u}_{*} \in L^{2}\left(\Omega, \mathbb{R}^{3 \times 3}\right)$ thus proving that $\mathbf{u}_{*} \in H^{1}\left(\Omega, \mathbb{R}^{3}\right)$.

Lemma 4.2 (Lower bound). Assume $(\mathcal{L} 1),(\mathcal{L} 2),(\mathcal{W} \mathbf{1}),(\mathcal{W} \mathbf{2}),(\mathcal{W} \mathbf{3}),(\mathcal{W} 4)$. Let $\left(\mathbf{y}_{j}\right)_{j \in \mathbb{N}} \subset$ $W^{1, p}\left(\Omega, \mathbb{R}^{3}\right)$ be a sequence. For any $j \in \mathbb{N}$, let $\mathbf{R}_{j} \in \mathcal{A}_{p}\left(\mathbf{y}_{j}\right)$ and $\mathbf{u}_{j}(\mathbf{x}):=h_{j}^{-1}\left(\mathbf{R}_{j}^{T} \mathbf{y}_{j}(\mathbf{x})-\right.$ $\mathbf{x})$. Suppose that there exists $\mathbf{u} \in W^{1, p}\left(\Omega, \mathbb{R}^{3}\right)$ such that $\nabla \mathbf{u}_{j} \rightarrow \nabla \mathbf{u}$ weakly in $L^{p}\left(\Omega, \mathbb{R}^{3 \times 3}\right)$. Then

$$
\liminf _{j \rightarrow+\infty} \mathcal{G}_{h_{j}}^{I}\left(\mathbf{y}_{j}\right) \geq \mathcal{G}^{I}(\mathbf{u})
$$

Proof We may assume wlog that $\mathcal{G}_{h_{j}}^{I}\left(\mathbf{y}_{j}\right) \leq M$ for any $j \in \mathbb{N}$ hence $\mathbf{u} \in H^{1}\left(\Omega ; \mathbb{R}^{3}\right)$ by Lemma (4.1) and

$$
\begin{aligned}
1 & =\operatorname{det} \nabla \mathbf{y}_{j}=\operatorname{det}\left(\mathbf{R}_{j}\left(\mathbf{I}+h_{j} \nabla \mathbf{u}_{j}\right)\right)=\operatorname{det}\left(\mathbf{I}+h_{j} \nabla \mathbf{u}_{j}\right)= \\
& =1+h_{j} \operatorname{div} \mathbf{u}_{j}-\frac{1}{2} h_{j}^{2}\left(\operatorname{Tr}\left(\nabla \mathbf{u}_{j}\right)^{2}-\left(\operatorname{Tr} \nabla \mathbf{u}_{j}\right)^{2}\right)+h_{j}^{3} \operatorname{det} \nabla \mathbf{u}_{j}
\end{aligned}
$$

a.e. in $\Omega$, that is,

$$
\operatorname{div} \mathbf{u}_{j}=\frac{1}{2} h_{j}\left(\operatorname{Tr}\left(\nabla \mathbf{u}_{j}\right)^{2}-\left(\operatorname{Tr} \nabla \mathbf{u}_{j}\right)^{2}\right)-h_{j}^{2} \operatorname{det} \nabla \mathbf{u}_{j} .
$$

By taking into account that $\nabla \mathbf{u}_{j}$ are uniformly bounded in $L^{p}$ we get $h_{j}^{\alpha}\left|\nabla \mathbf{u}_{j}\right| \rightarrow 0$ a.e. in $\Omega$ for every $\alpha>0$ hence $\operatorname{div} \mathbf{u}_{j}=\frac{1}{2} h_{j}\left(\operatorname{Tr}\left(\nabla \mathbf{u}_{j}\right)^{2}-\left(\operatorname{Tr} \nabla \mathbf{u}_{j}\right)^{2}\right)-h_{j}^{2} \operatorname{det} \nabla \mathbf{u}_{j} \rightarrow 0$ a.e. in $\Omega$. Since the weak convergence of $\nabla \mathbf{u}_{j}$ implies div $\mathbf{u}_{j} \rightarrow \operatorname{div} \mathbf{u}$ weakly in $L^{p}(\Omega)$ we get $\operatorname{div} \mathbf{u}=0$ a.e. in $\Omega$. By setting

$$
\mathbf{D}_{j}:=\mathbb{E}\left(\mathbf{u}_{j}\right)+\frac{1}{2} h_{j} \nabla \mathbf{u}_{j}^{T} \nabla \mathbf{u}_{j}
$$


by (3.3), (3.1) $(\mathcal{L} 1)$ and $(\mathcal{L} 2)$, and by recalling that $B_{j}$ is defined in (4.6), we get for large enough $j$

$$
\begin{aligned}
\mathcal{G}_{h_{j}}^{I}\left(\mathbf{v}_{j}\right) & \geq \frac{1}{h_{j}^{2}} \int_{B_{j}} \mathcal{V}\left(\mathbf{x}, h_{j} \mathbf{D}_{j}\right) d \mathbf{x}-\mathcal{L}\left(\mathbf{R}_{j} \mathbf{u}_{j}\right)-h_{j}^{-1} \mathcal{L}\left(\left(\mathbf{R}_{j}-\mathbf{I}\right) \mathbf{x}\right) \\
& \geq \int_{B_{j}} \frac{1}{2} \mathbf{D}_{j}^{T} D^{2} \mathcal{V}(\mathbf{x}, \mathbf{0}) \mathbf{D}_{j} d \mathbf{x}-\int_{B_{j}} \eta\left(h_{j} \mathbf{D}_{j}\right)\left|\mathbf{D}_{j}\right|^{2} d \mathbf{x}-\mathcal{L}\left(\mathbf{R}_{j} \mathbf{u}_{j}\right) \\
& \geq \frac{1}{2} \int_{\Omega}\left(\mathbf{1}_{B_{j}} \mathbf{D}_{j}\right)^{T} D^{2} \mathcal{W}(\mathbf{x}, \mathbf{I})\left(\mathbf{1}_{B_{j}} \mathbf{D}_{j}\right) d \mathbf{x}-\eta\left(\sqrt{h}_{j}\right) \int_{\Omega}\left|\mathbf{1}_{B_{j}} \mathbf{D}_{j}\right|^{2} d \mathbf{x}-\mathcal{L}\left(\mathbf{R}_{j} \mathbf{u}_{j}\right),
\end{aligned}
$$

since on $B_{j}$ we have $h_{j}\left|\mathbf{D}_{j}\right| \leq \sqrt{h_{j}}\left(\sqrt{h_{j}}\left|\nabla \mathbf{v}_{j}\right|+\frac{1}{2} h_{j}^{3 / 2}\left|\nabla \mathbf{v}_{j}^{T}\right|\left|\nabla \mathbf{v}_{j}\right|\right) \leq 2 \sqrt{h_{j}}$ for large enough $j$ (so that indeed (3.3) can be applied) and since $\eta$ is increasing. Since $h_{j} \nabla \mathbf{u}_{j}^{T} \nabla \mathbf{u}_{j} \rightarrow 0$ a.e. in $\Omega$ and $\left|B_{j}^{c}\right| \rightarrow 0$ as $j \rightarrow+\infty$, and since $\left|\mathbf{1}_{B_{j}} h_{j} \nabla \mathbf{u}_{j}^{T} \nabla \mathbf{u}_{j}\right| \leq 1$, we get $\mathbf{1}_{B_{j}} h_{j} \nabla \mathbf{u}_{j}^{T} \nabla \mathbf{u}_{j} \rightarrow 0$ weakly in $L^{2}\left(\Omega, \mathbb{R}^{3 \times 3}\right)$. By taking into account that $\mathbf{1}_{B_{j}} \nabla \mathbf{u}_{j} \rightarrow \nabla \mathbf{u}$ weakly in $L^{2}\left(\Omega, \mathbb{R}^{3 \times 3}\right)$, we then obtain $\mathbf{1}_{B_{j}} \mathbf{D}_{j} \rightarrow \mathbb{E}(\mathbf{u})$ weakly in $L^{2}\left(\Omega, \mathbb{R}^{3 \times 3}\right)$. Let now $\mathbf{c}_{j} \in \mathbb{R}^{3}$ such that $\mathbf{u}_{j}-\mathbf{c}_{j} \rightarrow \mathbf{u}$ weakly in $W^{1, p}\left(\Omega, \mathbb{R}^{3 \times 3}\right)$. By taking into account that, up to subsequences, Lemma 4.1 entails $\mathbf{R}_{j} \rightarrow \mathbf{R} \in \mathcal{S}_{\mathcal{L}}^{0}$, we get

$$
\lim _{j \rightarrow+\infty} \mathcal{L}\left(-\mathbf{R}_{j} \mathbf{u}_{j}\right)=\lim _{j \rightarrow+\infty} \mathcal{L}\left(-\mathbf{R}_{j}\left(\mathbf{u}_{j}-\mathbf{c}_{j}\right)\right)=-\mathcal{L}(\mathbf{R} \mathbf{u}) .
$$

Hence, by $(\mathcal{W} 3),(4.7)$ and by the weak $L^{2}\left(\Omega, \mathbb{R}^{3 \times 3}\right)$ lower semicontinuity of the map $\mathbf{F} \mapsto \int_{\Omega} \mathbf{F}^{T} D^{2} \mathcal{W}(\mathbf{x}, \mathbf{I}) \mathbf{F} d \mathbf{x}$, we conclude

$$
\liminf _{j \rightarrow+\infty} \mathcal{G}_{h_{j}}^{I}\left(\mathbf{v}_{j}\right) \geq \frac{1}{2} \int_{\Omega} \mathbb{E}(\mathbf{u}) D^{2} \mathcal{W}(\mathbf{x}, \mathbf{I}) \mathbb{E}(\mathbf{u}) d \mathbf{x}-\mathcal{L}(\mathbf{R u}) \geq \mathcal{G}^{I}(\mathbf{u})
$$

which ends the proof.

We next provide the construction for the recovery sequence, taking advantage of the following approximation result from [12].

Lemma 4.3 ( [12, Lemma 6.2]) Suppose that $\partial \Omega$ has a finite number of connected components. Let $\left(h_{j}\right)_{j \in \mathbb{N}} \subset(0,1)$ be a vanishing sequence. Let $\mathbf{u} \in H_{\mathrm{div}}^{1}\left(\Omega, \mathbb{R}^{3}\right)$. There exists a sequence $\left(\mathbf{u}_{j}\right)_{j \in \mathbb{N}} \subset W^{2, \infty}\left(\Omega, \mathbb{R}^{3}\right)$ such that

i) $\operatorname{det}\left(\mathbf{I}+h_{j} \nabla \mathbf{u}_{j}\right)=1$ for any $j \in \mathbb{N}$,

ii) $h_{j}\left\|\nabla \mathbf{u}_{j}\right\|_{L^{\infty}\left(\Omega, \mathbb{R}^{3 \times 3}\right)} \rightarrow 0$ as $j \rightarrow+\infty$,

iii) $\mathbf{u}_{j} \rightarrow \mathbf{u}$ strongly in $H^{1}\left(\Omega, \mathbb{R}^{3}\right)$ as $j \rightarrow+\infty$.

Lemma 4.4 (Upper bound). Suppose that $\partial \Omega$ has a finite number of connected compo-

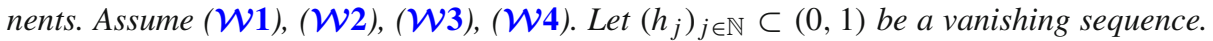
For every $\mathbf{u} \in W^{1, p}\left(\Omega, \mathbb{R}^{3}\right)$ there exists a sequence $\left(\mathbf{u}_{j}\right)_{j \in \mathbb{N}} \subset W^{1, p}\left(\Omega, \mathbb{R}^{3}\right)$ such that $\mathbf{u}_{j} \rightarrow \mathbf{u}$ weakly in $W^{1, p}\left(\Omega, \mathbb{R}^{3}\right)$ as $j \rightarrow+\infty$ and $\mathbf{R}_{*} \in \mathcal{S}_{\mathcal{L}}^{0}$ such that by setting $\mathbf{y}_{j}:=\mathbf{R}_{*}\left(\mathbf{x}+h_{j} \mathbf{u}_{j}\right)$ we have

$$
\limsup _{j \rightarrow+\infty} \mathcal{G}_{h_{j}}^{I}\left(\mathbf{y}_{j}\right) \leq \mathcal{G}^{I}(\mathbf{u}) .
$$

Proof It is enough to prove the result in case $\mathbf{u} \in H_{\text {div }}^{1}\left(\Omega, \mathbb{R}^{3}\right)$. We take the sequence $\left(\mathbf{u}_{j}\right)_{j \in \mathbb{N}}$ from Lemma 4.3 so that $\mathbf{u}_{j} \rightarrow \mathbf{u}$ strongly in $H^{1}\left(\Omega, \mathbb{R}^{3}\right)$ as $j \rightarrow+\infty$, and we take

$$
\mathbf{R}_{*} \in \operatorname{argmin}\left\{\int_{\Omega} \mathcal{Q}^{I}(\mathbf{x}, E(\mathbf{u})) d \mathbf{x}-\mathcal{L}(\mathbf{R u}): \mathbf{R} \in \mathcal{S}_{\mathcal{L}}^{0}\right\} .
$$


We set $\mathbf{y}_{j}:=\mathbf{R}_{*}\left(\mathbf{x}+h_{j} \mathbf{u}_{j}\right)$ and $\mathcal{F}\left(\mathbf{u}_{j}\right):=\frac{1}{2} \int_{\Omega} \nabla \mathbf{u}_{j}^{T} D^{2} \mathcal{W}(\mathbf{x}, \mathbf{I}) \nabla \mathbf{u}_{j} d \mathbf{x}-\mathcal{L}\left(\mathbf{R}_{*} \mathbf{u}_{j}\right)$ for any $j \in \mathbb{N}$. Property ii) of Lemma 4.3 yields $\mathbf{I}+h_{j} \nabla \mathbf{u}_{j} \in \mathcal{U}$ for a.e. $\mathbf{x}$ in $\Omega$ if $j$ is large enough, where $\mathcal{U}$ is the neighbor of $S O(3)$ that appears in $(\mathcal{W} 3)$. In particular, $D^{2} \mathcal{W}(\mathbf{x}, \cdot) \in C^{2}(\mathcal{U})$ for a.e. $\mathbf{x} \in \Omega$

and we make use of (3.2) together with $\operatorname{det}\left(\mathbf{I}+h_{j} \nabla \mathbf{u}_{j}\right)=1$ and $\mathbf{R}_{*} \in \mathcal{S}_{\mathcal{L}}^{0}$ to obtain

$$
\begin{aligned}
\limsup _{j \rightarrow+\infty}\left|\mathcal{G}_{h_{j}}^{I}\left(\mathbf{y}_{j}\right)-\mathcal{F}\left(\mathbf{u}_{j}\right)\right| & \leq \limsup _{j \rightarrow+\infty} \int_{\Omega}\left|\frac{1}{h_{j}^{2}} \mathcal{W}^{I}\left(\mathbf{x}, \mathbf{I}+h_{j} \nabla \mathbf{u}_{j}\right)-\frac{1}{2} \nabla \mathbf{u}_{j}^{T} D^{2} \mathcal{W}(\mathbf{x}, \mathbf{I}) \nabla \mathbf{u}_{j}\right| d \mathbf{x} \\
& =\limsup _{j \rightarrow+\infty} \int_{\Omega}\left|\frac{1}{h_{j}^{2}} \mathcal{W}\left(\mathbf{x}, \mathbf{I}+h_{j} \nabla \mathbf{u}_{j}\right)-\frac{1}{2} \nabla \mathbf{u}_{j}^{T} D^{2} \mathcal{W}(\mathbf{x}, \mathbf{I}) \nabla \mathbf{u}_{j}\right| d \mathbf{x} \\
& \leq \limsup _{j \rightarrow+\infty} \int_{\Omega} \omega\left(h_{j}\left|\nabla \mathbf{u}_{j}\right|\right)\left|\nabla \mathbf{u}_{j}\right|^{2} d \mathbf{x} \\
& \leq \limsup _{j \rightarrow+\infty}\left\|\omega\left(h_{j}\left|\nabla \mathbf{u}_{j}\right|\right)\right\|_{L^{\infty}(\Omega)} \int_{\Omega}\left|\nabla \mathbf{u}_{j}\right|^{2} d \mathbf{x}=0 .
\end{aligned}
$$

The limit in the last line is zero since $h_{j} \nabla \mathbf{u}_{j} \rightarrow 0$ in $L^{\infty}\left(\Omega, \mathbb{R}^{3 \times 3}\right)$, since $\omega$ is increasing with $\lim _{t \rightarrow 0^{+}} \omega(t) \rightarrow 0$ and since $\mathbf{u}_{j} \rightarrow \mathbf{u}$ in $H^{1}\left(\Omega, \mathbb{R}^{3}\right)$ as $j \rightarrow+\infty$. Then,

$$
\begin{aligned}
\limsup _{j \rightarrow+\infty}\left|\mathcal{G}_{h_{j}}^{I}\left(\mathbf{y}_{j}\right)-\mathcal{G}^{I}(\mathbf{u})\right| \leq & \limsup _{j \rightarrow+\infty}\left|\mathcal{G}_{h_{j}}^{I}\left(\mathbf{y}_{j}\right)-\mathcal{F}\left(\mathbf{u}_{j}\right)\right|+\limsup _{j \rightarrow+\infty}\left|\mathcal{F}\left(\mathbf{u}_{j}\right)-\mathcal{G}^{I}(\mathbf{u})\right| \\
\leq \limsup _{j \rightarrow+\infty}\left|\frac{1}{2} \int_{\Omega} \nabla \mathbf{u}_{j}^{T} D^{2} \mathcal{W}(\mathbf{x}, \mathbf{I}) \nabla \mathbf{u}_{j}-\frac{1}{2} \int_{\Omega} \nabla \mathbf{u}^{T} D^{2} \mathcal{W}(\mathbf{x}, \mathbf{I}) \nabla \mathbf{u}\right| & \quad+\limsup _{j \rightarrow+\infty}\left|\mathcal{L}\left(\mathbf{R}_{*} \mathbf{u}_{j}\right)-\mathcal{L}\left(\mathbf{R}_{*} \mathbf{u}\right)\right|=0
\end{aligned}
$$

where the limit is zero since $\mathbf{u}_{j} \rightarrow \mathbf{u}$ strongly in $H^{1}\left(\Omega, \mathbb{R}^{3}\right)$ as $j \rightarrow+\infty$.

We next conclude the proof of Theorem 2.5 after having recalled that functionals $\mathcal{G}_{h}^{I}$ are uniformly bounded from below, which is a result that is shown in [12].

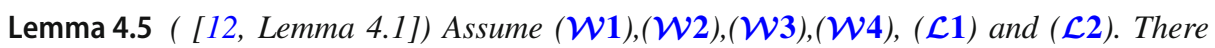
exists a constant $C>0$ (only depending on $\Omega, p, \mathbf{f}, \mathbf{g})$ such that $\mathcal{G}_{h}^{I}(\mathbf{y}) \geq-C$ for any $h \in(0,1)$ and any $\mathbf{y} \in W^{1, p}\left(\Omega, \mathbb{R}^{3}\right)$.

Proof of Theorem 2.5. We obtain (2.6) from Lemma 4.5. If $\left(\mathbf{y}_{j}\right)_{j \in \mathbb{N}} \subset W^{1, p}\left(\Omega, \mathbb{R}^{3}\right)$ is a sequence of quasi-minimizers of $\mathcal{G}_{h_{j}}^{I}$, then by Lemma 4.1 there exists $\mathbf{u}_{*} \in H^{1}\left(\Omega, \mathbb{R}^{3}\right)$ such that if $\mathbf{R}_{j} \in \mathcal{A}_{p}\left(\mathbf{y}_{j}\right)$ and $\mathbf{u}_{j}(\mathbf{x}):=h_{j}^{-1} \mathbf{R}_{j}^{T}\left(\mathbf{y}_{j}(\mathbf{x})-\mathbf{R}_{j} \mathbf{x}\right)$ then, up to subsequences, $\nabla \mathbf{u}_{j} \rightarrow \nabla \mathbf{u}_{*}$ weakly in $L^{p}(\Omega)$. Hence by Lemma 4.2

$$
\liminf _{j \rightarrow+\infty} \mathcal{G}_{h_{j}}^{I}\left(\mathbf{y}_{j}\right) \geq \mathcal{G}^{I}\left(\mathbf{u}_{*}\right) .
$$

On the other hand, by Lemma 4.4 , for every $\mathbf{u} \in W^{1, p}\left(\Omega, \mathbb{R}^{3}\right)$ there exist a sequence $\left(\mathbf{u}_{j}\right)_{j \in \mathbb{N}} \subset W^{1, p}\left(\Omega, \mathbb{R}^{3}\right)$ satisfying $\mathbf{u}_{j} \rightarrow \mathbf{u}$ weakly in $W^{1, p}\left(\Omega, \mathbb{R}^{3}\right)$ as $j \rightarrow+\infty$ and $\mathbf{R}_{*} \in$ $\mathcal{S}_{\mathcal{L}}^{0}$ such that by setting $\tilde{\mathbf{y}}_{j}:=\mathbf{R}_{*}\left(\mathbf{x}+h_{j} \mathbf{u}_{j}\right)$ we have

$$
\limsup _{j \rightarrow+\infty} \mathcal{G}_{h_{j}}^{I}\left(\tilde{\mathbf{y}}_{j}\right) \leq \mathcal{G}^{I}(\mathbf{u}) \text {. }
$$

Since

$$
\mathcal{G}_{h_{j}}^{I}\left(\mathbf{y}_{j}\right)+o(1)=\inf _{W^{1, p}\left(\Omega, \mathbb{R}^{3}\right)} \mathcal{G}_{h_{j}}^{I} \leq \mathcal{G}_{h_{j}}^{I}\left(\tilde{\mathbf{y}}_{j}\right) \quad \text { as } j \rightarrow+\infty
$$


by passing to the limit as $j \rightarrow+\infty$ we get $\mathcal{G}^{I}\left(\mathbf{u}_{*}\right) \leq \mathcal{G}^{I}(\mathbf{u})$ for every $\mathbf{u} \in H^{1}\left(\Omega, \mathbb{R}^{3}\right)$ thus completing the proof.

\subsection{The compressible case}

Here we briefly show how to adapt the previous arguments to obtain the proof of Theorem 2.4.

Lemma 4.6 (Compactness). Assume $(\mathcal{W} 1),(\mathcal{W} 2),(\mathcal{W} 3),(\mathcal{W} 4),(\mathcal{L} 1)$ and $(\mathcal{L} 2)$. Let $\left(h_{j}\right)_{j \subset \mathbb{N}} \subset$ $(0,1)$ be a vanishing a sequence, let $M>0$ and let $\left(\mathbf{y}_{j}\right)_{j \in \mathbb{N}} \subset W^{1, p}\left(\Omega, \mathbb{R}^{3}\right)$ be a sequence such that

$$
\mathcal{G}_{h_{j}}\left(\mathbf{y}_{j}\right) \leq M \quad \forall j \in \mathbb{N} .
$$

Let $\mathbf{R}_{j} \in \mathcal{A}_{p}\left(\mathbf{y}_{j}\right)$ and $\mathbf{u}_{j}(\mathbf{x}):=h_{j}^{-1}\left(\mathbf{R}_{j}^{T} \mathbf{y}_{j}(\mathbf{x})-\mathbf{x}\right)$. Then, the sequence $\left(\nabla \mathbf{u}_{j}\right)_{j \in \mathbb{N}}$ is bounded in $L^{p}\left(\Omega, \mathbb{R}^{3 \times 3}\right)$ and any of its weak $L^{p}\left(\Omega, \mathbb{R}^{3 \times 3}\right)$ limit points is of the form $\nabla \mathbf{u}_{*}$ for some $\mathbf{u}_{*} \in H^{1}\left(\Omega, \mathbb{R}^{3}\right)$. Moreover, any limit point of the sequence $\left(\mathbf{R}_{j}\right)_{j \in \mathbb{N}} \subset S O$ (3) belongs to $\mathcal{S}_{\mathcal{L}}^{0}$

Proof It is readily seen that inequalities (4.2) and (4.3) holds true with $\mathcal{W}$ in place of $\mathcal{W}^{I}$ hence by arguing as in Lemma 4.1 we get that $\nabla \mathbf{u}_{j}$ are equibounded in $L^{p}$. Moreover (4.8) entails the analogous of (4.5) hence $\mathcal{L}\left(\left(\mathbf{R}_{j}-\mathbf{I}\right) \mathbf{x}\right) \rightarrow 0$ as $j \rightarrow+\infty$ and if $\mathbf{R}_{j} \rightarrow \mathbf{R}_{*}$ along a suitable subsequence, we have $\mathcal{L}\left(\left(\mathbf{R}_{*}-\mathbf{I}\right) \mathbf{x}\right)=0$ that is $\mathbf{R}_{*} \in \mathcal{S}_{\mathcal{L}}^{0}$. The remaining part of the proof is identical to that of Lemma 4.1.

Lemma 4.7 (Lower bound). Assume $(\mathcal{L} 1),(\mathcal{L} 2),(\mathcal{W} 1),(\mathcal{W} \mathbf{2}),(\mathcal{W} 3),(\mathcal{W} 4)$. Let $\left(\mathbf{y}_{j}\right)_{j \in \mathbb{N}} \subset$ $W^{1, p}\left(\Omega, \mathbb{R}^{3}\right)$ be a sequence. For any $j \in \mathbb{N}$, let $\mathbf{R}_{j} \in \mathcal{A}_{p}\left(\mathbf{y}_{j}\right)$ and $\mathbf{u}_{j}(\mathbf{x}):=h_{j}^{-1} \mathbf{R}_{j}^{T}\left(\mathbf{y}_{j}(\mathbf{x})-\right.$ $\left.\mathbf{R}_{j} \mathbf{x}\right)$. Suppose that there exists $\mathbf{u} \in W^{1, p}\left(\Omega, \mathbb{R}^{3}\right)$ such that $\nabla \mathbf{u}_{j} \rightarrow \nabla \mathbf{u}$ weakly in $L^{p}\left(\Omega, \mathbb{R}^{3}\right)$. Then

$$
\liminf _{j \rightarrow+\infty} \mathcal{G}_{h_{j}}\left(\mathbf{y}_{j}\right) \geq \mathcal{G}(\mathbf{u}) .
$$

Proof It is enough to notice that inequality (4.7) in the proof of Lemma 4.2 holds true with $\mathcal{G}_{h_{j}}$ in place of $\mathcal{G}_{h_{j}}^{I}$. The proof follows by means of the same arguments therein.

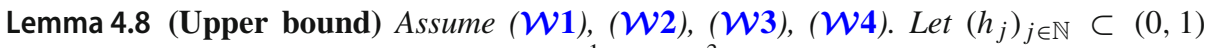
be a vanishing sequence. For every $\mathbf{u} \in W^{1, p}\left(\Omega, \mathbb{R}^{3}\right)$ there exists a sequence $\left(\mathbf{u}_{j}\right)_{j \in \mathbb{N}} \subset$ $W^{1, p}\left(\Omega, \mathbb{R}^{3}\right)$ such that $\mathbf{u}_{j} \rightarrow \mathbf{u}$ weakly in $W^{1, p}\left(\Omega, \mathbb{R}^{3}\right)$ as $j \rightarrow+\infty$ and $\mathbf{R}_{*} \in \mathcal{S}_{\mathcal{L}}^{0}$ such that by setting $\mathbf{y}_{j}:=\mathbf{R}_{*}\left(\mathbf{x}+h_{j} \mathbf{u}_{j}\right)$ we have

$$
\limsup _{j \rightarrow+\infty} \mathcal{G}_{h_{j}}\left(\mathbf{y}_{j}\right) \leq \mathcal{G}(\mathbf{u}) .
$$

Proof We assume wlog that $\mathbf{u} \in H^{1}\left(\Omega, \mathbb{R}^{3}\right)$. If we let $\left(\mathbf{u}_{j}\right)_{j \in \mathbb{N}}$ be a sequence obtained by a standard mollification of $\mathbf{u}$, then properties ii) and iii) of Lemma 4.3 hold true. We also let

$$
\mathbf{R}_{*} \in \operatorname{argmin}\left\{\int_{\Omega} \mathcal{Q}(\mathbf{x}, E(\mathbf{u})) d \mathbf{x}-\mathcal{L}(\mathbf{R u}): \mathbf{R} \in \mathcal{S}_{\mathcal{L}}^{0}\right\},
$$

so that by letting $\mathbf{y}_{j}:=\mathbf{R}_{*}\left(\mathbf{x}+h_{j} \mathbf{u}_{j}\right)$ we obtain

$$
\begin{aligned}
& \limsup _{j \rightarrow+\infty}\left|\mathcal{G}_{h_{j}}\left(\mathbf{y}_{j}\right)-\mathcal{G}\left(\mathbf{u}_{j}\right)\right| \\
& \quad \leq \limsup _{j \rightarrow+\infty} \int_{\Omega}\left|\frac{1}{h_{j}^{2}} \mathcal{W}\left(\mathbf{x}, \mathbf{I}+h_{j} \nabla \mathbf{u}_{j}\right)-\frac{1}{2} \nabla \mathbf{u}_{j}^{T} D^{2} \mathcal{W}(\mathbf{x}, \mathbf{I}) \nabla \mathbf{u}_{j}\right| d \mathbf{x}=0
\end{aligned}
$$


where the limit is zero by the same argument used in the proof of Lemma 4.4. Therefore

$$
\begin{aligned}
& \limsup _{j \rightarrow+\infty}\left|\mathcal{G}_{h_{j}}\left(\mathbf{y}_{j}\right)-\mathcal{G}(\mathbf{u})\right| \leq \limsup _{j \rightarrow+\infty}\left|\mathcal{G}_{h_{j}}\left(\mathbf{y}_{j}\right)-\mathcal{G}\left(\mathbf{u}_{j}\right)\right|+\limsup _{j \rightarrow+\infty}\left|\mathcal{G}\left(\mathbf{u}_{j}\right)-\mathcal{G}(\mathbf{u})\right| \\
& \leq \limsup _{j \rightarrow+\infty}\left|\frac{1}{2} \int_{\Omega} \nabla \mathbf{u}_{j}^{T} D^{2} \mathcal{W}(\mathbf{x}, \mathbf{I}) \nabla \mathbf{u}_{j}-\frac{1}{2} \int_{\Omega} \nabla \mathbf{u}^{T} D^{2} \mathcal{W}(\mathbf{x}, \mathbf{I}) \nabla \mathbf{u}\right| \\
& \quad+\limsup _{j \rightarrow+\infty}\left|\mathcal{L}\left(\mathbf{R}_{*} \mathbf{u}_{j}\right)-\mathcal{L}\left(\mathbf{R}_{*} \mathbf{u}\right)\right|=0
\end{aligned}
$$

where the limit is zero thanks to the strong convergence of $\mathbf{u}_{j}$ to $\mathbf{u}$ in $H^{1}\left(\Omega, \mathbb{R}^{3}\right)$.

Proof of Theorem 2.4. By arguing as in Lemma 3.1 of [10] it is readily seen that there exists a constant $C>0$ (only depending on $\Omega, p, \mathbf{f}, \mathbf{g}$ ) such that $\mathcal{G}_{h}(\mathbf{y}) \geq-C$ for any $h \in(0,1)$ and any $\mathbf{y} \in W^{1, p}\left(\Omega, \mathbb{R}^{3}\right)$. Therefore the proof can be achieved by repeating the argument of the proof of Theorem 2.5 .

\section{The gap with linear elasticity: Proof of Theorem 2.7}

In this section we show that if $\mathcal{S}_{\mathcal{L}}^{0}$ is not reduced to the identity matrix, the minimization problem in the definition of functional $\mathcal{G}^{I}(\mathbf{u})($ resp. $\mathcal{G}(\mathbf{u}))$ is not solved in general by $\mathbf{R}=\mathbf{I}$ if $\mathbf{u}$ minimizes $\mathcal{G}^{I}$ (resp. $\mathcal{G}$ ) over $W^{1, p}\left(\Omega, \mathbb{R}^{3}\right.$ ). In particular, the minimal value of $\mathcal{G}^{I}$ (resp. $\mathcal{G})$ can be strictly below the minimum value of the standard functional of linearized elasticity $\mathcal{E}^{I}$ (resp. $\mathcal{E}$ ).

Here and in the following of this section, $\Omega$ is the set defined in (2.10), $B$ denotes the unit ball centered at the origin in the $x y$ plane, while $\nabla$ and $\Delta$ shall denote the gradient and the Laplacian in the $x, y$ variables, respectively. Moreover, the form of external forces is that of (2.11), and the conditions (f1)-(f2) are assumed to hold. We also introduce the auxiliary volume force field

$$
\tilde{\mathbf{f}}(x, y, z):=\left(\varphi_{y}(x, y),-\varphi_{x}(x, y), \psi(z)\right),
$$

and we notice that $\tilde{\mathbf{f}}=\tilde{\mathbf{R}} \mathbf{f}$, where $\tilde{\mathbf{R}}$ is the rotation matrix

$$
\tilde{\mathbf{R}}:=\left(\begin{array}{ccc}
0 & 1 & 0 \\
-1 & 0 & 0 \\
0 & 0 & 1
\end{array}\right)
$$

Exploiting (f1) and (f2), it is not difficult to check that $\mathcal{L}$ from (2.11) satisfies $(\mathcal{L} \mathbf{1})$. Concerning $(\mathcal{L} 2)$, in view of the general form of $\mathbf{W} \in \mathbb{R}_{\text {Skew }}^{3 \times 3}$, i.e.,

$$
\mathbf{W}:=\left(\begin{array}{ccc}
0 & a & b \\
-a & 0 & c \\
-b & -c & 0
\end{array}\right) \quad a, b, c \in \mathbb{R}
$$

under assumptions (f1) and (f2) we have

$$
\int_{\Omega} \mathbf{f}(\mathbf{x}) \cdot \mathbf{W} \mathbf{x} d \mathbf{x}=\int_{\Omega} \tilde{\mathbf{f}}(\mathbf{x}) \cdot \mathbf{W} \mathbf{x} d \mathbf{x}=0
$$

and

$$
\int_{\Omega} \mathbf{f}(\mathbf{x}) \cdot \mathbf{W}^{2} \mathbf{x} d \mathbf{x}=\int_{\Omega} \tilde{\mathbf{f}}(\mathbf{x}) \cdot \mathbf{W}^{2} \mathbf{x} d \mathbf{x}=-\pi\left(b^{2}+c^{2}\right) \int_{0}^{1} z \psi(z) d z \leq 0,
$$


so that by invoking the Euler-Rodrigues formula (2.1) we see that $\mathcal{L}$ satisfies $(\mathcal{L} 2)$ as well. From (5.2) we see that if $\int_{0}^{1} z \psi(z) d z>0$, then

$$
\int_{\Omega} \mathbf{f}(\mathbf{x}) \cdot \mathbf{W}^{2} \mathbf{x} d \mathbf{x}=0
$$

if and only if $b^{2}+c^{2}=0$, so that in view of (2.1) the set $\mathcal{S}_{\mathcal{L}}^{0}$ coincides with the set of rotation matrices around the $z$ axis, i.e.,

$$
\mathcal{S}_{\mathcal{L}}^{0}=\left\{\mathbf{R}_{\theta}: \theta \in[-\pi, \pi]\right\}, \quad \text { where } \quad \mathbf{R}_{\theta}=\left(\begin{array}{ccc}
\cos \theta & \sin \theta & 0 \\
-\sin \theta & \cos \theta & 0 \\
0 & 0 & 1
\end{array}\right),
$$

in particular $\mathcal{S}_{\mathcal{L}}^{0}$ is not reduced to the identity matrix and it is a strict subset of $S O(3)$.

On the other hand, if $\int_{0}^{1} z \psi(z) d z=0$, then $\mathcal{S}_{\mathcal{L}}^{0} \equiv S O(3)$.

In both cases we clearly have

$$
\tilde{\mathbf{R}} \in \mathcal{S}_{\mathcal{L}}^{0} \quad \text { and } \quad \tilde{\mathbf{R}}^{T} \in \mathcal{S}_{\mathcal{L}}^{0} .
$$

Concerning the strain energy density, in this section we assume that $\mathcal{W}$ satisfies $(\mathcal{W} \mathbf{1})$, $(\mathcal{W} 2),(\mathcal{W} 3),(\mathcal{W} 4)$ and (2.12), and we let $\mathcal{W}^{I}(\mathbf{x}, \mathbf{F})=\mathcal{W}^{I}(\mathbf{F})$ be equal to $\mathcal{W}(\mathbf{F})$ if $\operatorname{det} \mathbf{F}=1$ and equal to $+\infty$ otherwise. With these assumptions on $\mathcal{W}$, the functional of linearized elasticity is reduced to

$$
\mathcal{E}(\mathbf{u}):=4 \int_{\Omega}|\mathbb{E}(\mathbf{u})|^{2} d \mathbf{x}-\mathcal{L}(\mathbf{u}), \quad \mathbf{u} \in H^{1}\left(\Omega, \mathbb{R}^{3}\right),
$$

while the limit functional $\mathcal{G}$ becomes

$$
\mathcal{G}(\mathbf{u})=4 \int_{\Omega}|\mathbb{E}(\mathbf{u})|^{2} d \mathbf{x}-\mathcal{L}(\mathbf{u})-\max _{\mathbf{R} \in \mathcal{S}_{\mathcal{L}}^{0}} \mathcal{L}((\mathbf{R}-\mathbf{I}) \mathbf{x}), \quad \mathbf{u} \in H^{1}\left(\Omega, \mathbb{R}^{3}\right) .
$$

For the following arguments, it is also convenient to introduce the auxiliary functional

$$
\tilde{\mathcal{G}}(\mathbf{u}):=4 \int_{\Omega}|\mathbb{E}(\mathbf{u})|^{2} d \mathbf{x}-\mathcal{L}\left(\tilde{\mathbf{R}}^{T} \mathbf{u}\right), \quad \mathbf{u} \in H^{1}\left(\Omega, \mathbb{R}^{3}\right)
$$

where $\tilde{\mathbf{R}}$ is given by (5.1). The above functionals $\mathcal{E}, \mathcal{G}, \tilde{\mathcal{G}}$ are extended as usual to $W^{1, p}\left(\Omega, \mathbb{R}^{3}\right) \backslash H^{1}\left(\Omega, \mathbb{R}^{3}\right)$ with value $+\infty$. Due to $(\mathcal{L} \mathbf{1})$ and Korn inequality, it follows from standard arguments that the functionals $\mathcal{E}, \mathcal{G}, \tilde{\mathcal{G}}$ admit minimizers over $H^{1}\left(\Omega, \mathbb{R}^{3}\right)$.

We shall also consider the incompressible case, by considering functionals $\mathcal{G}^{I}, \mathcal{E}^{I}$, as defined in Sect. 2, under the assumption (2.12) for $\mathcal{W}$, and with external loads given by functional $\mathcal{L}$ from (2.3) with $\mathbf{g} \equiv 0$ and $\mathbf{f}$ of the form (2.11). Again, we introduce the auxiliary functional

$$
\tilde{\mathcal{G}}^{I}(\mathbf{u}):= \begin{cases}4 \int_{\Omega}|\mathbb{E}(\mathbf{u})|^{2} d \mathbf{x}-\mathcal{L}\left(\tilde{\mathbf{R}}^{T} \mathbf{u}\right) & \text { if } \mathbf{u} \in H_{\mathrm{div}}^{1}\left(\Omega, \mathbb{R}^{3}\right) \\ +\infty & \text { otherwise in } W^{1, p}\left(\Omega, \mathbb{R}^{3}\right) .\end{cases}
$$

where $\tilde{\mathbf{R}}$ is given by (5.1). Existence of minimizers also holds for $\mathcal{E}^{I}, \mathcal{G}^{I}, \tilde{\mathcal{G}}^{I}$, since the divergence-free constraint is weakly closed in $H^{1}\left(\Omega, \mathbb{R}^{3}\right)$.

Before proving Theorem 2.7, we provide an auxiliary statement. 
Lemma 5.1 Assume (2.10), (2.11), (2.12), (f1), (f2). Then there hold

$$
\begin{aligned}
\min _{H^{1}\left(\Omega, \mathbb{R}^{3}\right)} \tilde{\mathcal{G}} \leq & \min _{u \in H^{2}(B)} \int_{B} 8 u_{x y}^{2}+2\left(u_{y y}-u_{x x}\right)^{2}+\int_{B} u \Delta \varphi+\min _{w \in H^{1}(0,1)} 4 \pi \int_{0}^{1} w^{\prime 2} \\
& -\pi \int_{0}^{1} w \psi<0
\end{aligned}
$$

and

$$
\min _{H^{1}\left(\Omega, \mathbb{R}^{3}\right)} \tilde{\mathcal{G}}^{I} \leq \min _{u \in H^{2}(B)} \int_{B} 8 u_{x y}^{2}+2\left(u_{y y}-u_{x x}\right)^{2}+\int_{B} u \Delta \varphi<0 .
$$

Proof Let us prove the first statement.

Let $\mathcal{K} \subset H^{1}\left(\Omega, \mathbb{R}^{3}\right)$ be the class of displacement fields $\mathbf{v} \in H^{1}\left(\Omega, \mathbb{R}^{3}\right)$ of the form

$$
\mathbf{v}(x, y, z)=\left(u_{y}(x, y),-u_{x}(x, y), w(z)\right),
$$

for some $u \in H^{2}(\Omega)$ and some $w \in H^{1}(0,1)$. It is easily seen that $\mathcal{K}$ is weakly closed in $H^{1}\left(\Omega, \mathbb{R}^{3}\right)$. In particular, there are minimizers of $\tilde{\mathcal{G}}$ over $\mathcal{K}$. We notice that by means of (5.6) any couple $u \in H^{2}(B), w \in H^{1}(0,1)$ uniquely determines $\mathbf{v} \in \mathcal{K}$. Conversely, any $\mathbf{v} \in \mathcal{K}$ uniquely determines $u \in H^{2}(B)$, up to an additive constant, and $w \in H^{1}(0,1)$. Moreover a computation shows that the energy functional $\tilde{\mathcal{G}}$ takes the following form for any $\mathbf{v} \in \mathcal{K}$ :

$$
\begin{aligned}
\tilde{\mathcal{G}}(\mathbf{v})= & \tilde{\mathcal{G}}\left(\left(u_{y},-u_{x}, w\right)\right)=\int_{B} 8 u_{x y}^{2}+2\left(u_{y y}-u_{x x}\right)^{2}-\int_{B}\left(u_{y},-u_{x}\right) \cdot\left(\varphi_{y},-\varphi_{x}\right) \\
& +4 \pi \int_{0}^{1} w^{\prime 2} d z-\pi \int_{0}^{1} w \psi d z \\
= & \int_{B} 8 u_{x y}^{2}+2\left(u_{y y}-u_{x x}\right)^{2}+\int_{B} u \Delta \varphi+4 \pi \int_{0}^{1} w^{\prime 2} d z-\pi \int_{0}^{1} w \psi d z,
\end{aligned}
$$

having exploited the fact that $\nabla \varphi \cdot \mathbf{n}=0$ on $\partial B$, where $\mathbf{n}$ is the outer unit normal to $\partial B$. We deduce

$$
\begin{aligned}
\min _{H^{1}\left(\Omega, \mathbb{R}^{3}\right)} \tilde{\mathcal{G}} \leq \min _{\mathcal{K}} \tilde{\mathcal{G}}= & \min _{u \in H^{2}(B)} \int_{B} 8 u_{x y}^{2}+2\left(u_{y y}-u_{x x}\right)^{2}+\int_{B} u \Delta \varphi \\
& +\min _{w \in H^{1}(0,1)} 4 \pi \int_{0}^{1} w^{\prime 2}-\pi \int_{0}^{1} w \psi,
\end{aligned}
$$

where the last minimization problem in (5.7) has a solution as well, thanks to $\int_{\Omega} \psi=0$ from (f2) and to Poincaré inequality.

If we introduce the perturbation $u+\varepsilon \zeta$, where $\varepsilon>0$ and $\zeta \in H_{0}^{2}(B)$, we get the first order optimality condition for the first minimization problem in the right hand side of (5.7)

$$
4 \int_{B} 4 u_{x y} \zeta_{x y}+u_{y y} \zeta_{y y}+u_{x x} \zeta_{x x}-u_{y y} \zeta_{x x}-u_{y y} \zeta_{x x}=-\int_{B} \zeta \Delta \varphi,
$$

and after integration by parts we obtain the Euler-Lagrange equation

$$
4 \Delta^{2} u+\Delta \varphi=0 \quad \text { in } \mathcal{D}^{\prime}(B),
$$

where $\Delta^{2}$ denotes the planar biharmonic operator.

In view of (5.7), and since $\tilde{\mathcal{G}}(\mathbf{0})=0$, in order to conclude it is enough to show that $\min _{\mathcal{K}} \tilde{\mathcal{G}} \neq 0$. Assume by contradiction that $\min _{\mathcal{K}} \tilde{\mathcal{G}}=0$ : then $u \equiv 0$ on $B, w \equiv 0$ on $(0,1)$ are solutions to the minimization problems in the right hand side of $(5.7)$, so that 
$\Delta^{2} u \equiv 0$ in $B$ and from above the Euler-Lagrange equation we deduce $\Delta \varphi \equiv 0$ in $B$. This is a contradiction with assumption (f) 1 .

In order to prove the second statement, we consider the subset $\mathcal{K}_{\text {div }}$ of $H_{\text {div }}^{1}\left(\Omega, \mathbb{R}^{3}\right)$ made of vector fields of the form $\mathbf{v}(x, y, z)=\left(u_{y}(x, y),-u_{x}(x, y), 0\right)$ for some $u \in H^{2}(B)$, and for any $\mathbf{v} \in \mathcal{K}_{\text {div }}$ the energy functional $\tilde{\mathcal{G}}^{I}$ has the expression

$$
\tilde{\mathcal{G}}^{I}(\mathbf{v})=\tilde{\mathcal{G}}^{I}\left(\left(u_{y},-u_{x}, 0\right)\right)=\int_{B} 8 u_{x y}^{2}+2\left(u_{y y}-u_{x x}\right)^{2}+\int_{B} u \Delta \varphi,
$$

so that

$$
\min _{H^{1}\left(\Omega, \mathbb{R}^{3}\right)} \tilde{\mathcal{G}}^{I} \leq \min _{\mathcal{K}_{\mathrm{div}}} \tilde{\mathcal{G}}^{I}=\min _{u \in H^{2}(B)} \int_{B} 8 u_{x y}^{2}+2\left(u_{y y}-u_{x x}\right)^{2}+\int_{B} u \Delta \varphi
$$

and the proof concludes with the same argument as above.

We proceed to the proof of Theorem 2.7.

Proof of Theorem 2.7. We will prove that there holds

$$
\min _{H^{1}\left(\Omega, \mathbb{R}^{3}\right)} \mathcal{G} \leq \min _{H^{1}\left(\Omega, \mathbb{R}^{3}\right)} \tilde{\mathcal{G}}<\min _{H^{1}\left(\Omega, \mathbb{R}^{3}\right)} \mathcal{E},
$$

and that if $\|\psi\|_{L^{2}(\Omega)}$ is small enough there also holds

$$
\min _{H^{1}\left(\Omega, \mathbb{R}^{3}\right)} \mathcal{G}^{I} \leq \min _{H^{1}\left(\Omega, \mathbb{R}^{3}\right)} \tilde{\mathcal{G}}^{I}<\min _{H^{1}\left(\Omega, \mathbb{R}^{3}\right)} \mathcal{E}^{I} .
$$

We start by noticing that the first inequality in both statements is trivial, due to (5.4). Therefore, we are left to prove the second inequality in both cases.

Let $\mathbf{v}=\left(v_{1}, v_{2}, v_{3}\right) \in H^{1}\left(\Omega, \mathbb{R}^{3}\right)$. Let $\tilde{\mathbf{v}}:=\left(v_{1}, v_{2}\right)$, so that $\tilde{\mathbf{v}} \in H^{1}\left(\Omega, \mathbb{R}^{2}\right)$. Let $\tilde{\mathbf{u}} \in H^{1}\left(B, \mathbb{R}^{2}\right)$ be defined by $\tilde{\mathbf{u}}(x, y):=\int_{0}^{1} \tilde{\mathbf{v}}(x, y, z) d z$. Let moreover $\tilde{w} \in H^{1}(0,1)$ be defined by $\tilde{w}(z):=\frac{1}{\pi} \int_{B} v_{3}(x, y, z) d x d y$. Since $|\mathbb{E}(\mathbf{v})| \geq|\widetilde{\mathbb{E}}(\mathbf{v})|=|\widetilde{\mathbb{E}}(\tilde{\mathbf{v}})|$, where $\widetilde{\mathbb{E}}(\cdot)$ is the upper-left $2 \times 2$ submatrix of $\mathbb{E}(\cdot)$, by Jensen inequality we have

$$
\begin{aligned}
\mathcal{E}^{I}(\mathbf{v}) \geq & \mathcal{E}(\mathbf{v}) \geq 4 \int_{\Omega}|\mathbb{E}(\mathbf{v})|^{2}-\int_{\Omega}\left(\varphi_{x}, \varphi_{y}, \psi\right) \cdot \mathbf{v} \\
\geq & 4 \int_{B}\left|\int_{0}^{1} \widetilde{\mathbb{E}}(\tilde{\mathbf{v}}) d z\right|^{2} d x d y+4 \pi \int_{0}^{1}\left|\frac{1}{\pi} \int_{B} v_{3, z} d x d y\right|^{2} d z \\
& -\int_{B}\left(\varphi_{x}, \varphi_{y}\right) \cdot\left(\int_{0}^{1} \tilde{\mathbf{v}} d z\right) d x d y-\int_{0}^{1} \psi(z)\left(\int_{B} v_{3}(x, y, z) d x d y\right) d z \\
\geq & \mathcal{J}(\tilde{\mathbf{u}})+4 \pi \int_{0}^{1} \tilde{w}^{\prime 2}-\pi \int_{0}^{1} \tilde{w} \psi,
\end{aligned}
$$

where we have used the notation $v_{i, a}:=\partial_{a} v_{i}, i \in\{1,2,3\}, a \in\{x, y, z\}$, and where

$$
\mathcal{J}(\widetilde{\mathbf{u}}):=4 \int_{B}|\widetilde{\mathbb{E}}(\tilde{\mathbf{u}})|^{2}-\int_{B} \nabla \varphi \cdot \tilde{\mathbf{u}} .
$$

We claim that functional $\mathcal{J}$ admits a minimizer over $H^{1}\left(B, \mathbb{R}^{2}\right)$ which is the gradient of a $H^{2}(B)$ function. Indeed, thanks to (f1) and Korn inequality, a minimizer exists and it is unique up to planar infinitesimal rigid displacements. Moreover, thanks to a first variation argument, it is a solution to the boundary value problem

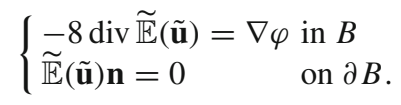


We claim that problem (5.10) admits a solution $\tilde{\mathbf{u}} \in H^{1}\left(B, \mathbb{R}^{2}\right)$ of the form $\tilde{\mathbf{u}}(x, y):=$ $r^{-1} \eta(r)(x, y)$, where $r:=\sqrt{x^{2}+y^{2}}$ (since $\tilde{\mathbf{u}} \in H^{1}\left(B, \mathbb{R}^{2}\right)$, we necessarily have $\left.\eta(0)=0\right)$. Indeed, a direct calculation shows that $\tilde{\mathbf{u}}(x, y)=r^{-1} \eta(r)(x, y)$ is a $H^{1}\left(B, \mathbb{R}^{2}\right)$ solution to problem (5.10) if and only if

$$
\left\{\begin{array}{l}
r^{2} \eta^{\prime \prime}+r \eta^{\prime}-\eta=-\frac{1}{8} r^{2} \phi^{\prime}(r) \text { in }(0,1) \\
\eta(0)=0 \\
\eta^{\prime}(1)=0
\end{array}\right.
$$

where $\phi$ is the radial profile of $\varphi$. Problem (5.11) has indeed a solution, whose explicit form is

$$
\eta_{*}(r)=-\frac{1}{16} r \phi(r)+\frac{1}{16 r} \int_{0}^{r} t^{2} \phi^{\prime}(t) d t .
$$

Therefore, letting $\Phi: B \rightarrow \mathbb{R}$ be the radial function defined by $\Phi(x, y):=\int_{0}^{r} \eta_{*}(t) d t$, we obtain $\Phi \in H^{2}(B)$ and moreover $\nabla \Phi=r^{-1} \eta(r)(x, y)$ solves (5.10), hence it minimizes $\mathcal{J}$ over $H^{1}\left(\Omega, \mathbb{R}^{2}\right)$. The claim is proved.

Therefore, the estimate (5.8) rewrites as

$$
\begin{aligned}
\mathcal{E}^{I}(\mathbf{v}) \geq \mathcal{E}(\mathbf{v}) & \geq \mathcal{J}(\tilde{\mathbf{u}})+4 \pi \int_{0}^{1} \tilde{w}^{\prime 2}-\pi \int_{0}^{1} \tilde{w} \psi \\
& \geq \min _{\Phi \in H^{2}(B)} 4 \int_{B}|\widetilde{\mathbb{E}}(\nabla \Phi)|^{2}-\int_{B} \nabla \Phi \cdot \nabla \varphi+4 \pi \int_{0}^{1} \tilde{w}^{\prime 2}-\pi \int_{0}^{1} \tilde{w} \psi
\end{aligned}
$$

Integrating by parts, since (f1) yields $\phi=0$ on $\partial B$, we get

$$
\mathcal{E}^{I}(\mathbf{v}) \geq \mathcal{E}(\mathbf{v}) \geq \min _{\Phi \in H^{2}(B)} 4 \int_{B}\left|D^{2} \Phi\right|^{2}+\int_{B} \Phi \Delta \varphi+4 \pi \int_{0}^{1} \tilde{w}^{\prime 2}-\pi \int_{0}^{1} \tilde{w} \psi,
$$

where $D^{2}$ denotes the Hessian in the $x, y$ variables, hence

$$
\begin{aligned}
\min _{H^{1}\left(\Omega, \mathbb{R}^{3}\right)} \mathcal{E}^{I} \geq \min _{H^{1}\left(\Omega, \mathbb{R}^{3}\right)} \mathcal{E} \geq & \min _{\Phi \in H^{2}(B)} \int_{B} 8 \Phi_{x y}^{2}+4 \Phi_{x x}^{2}+4 \Phi_{y y}^{2}+\int_{B} \Phi \Delta \varphi \\
& +\min _{w \in H^{1}(0,1)} 4 \pi \int_{0}^{1} w^{\prime 2}-\pi \int_{\Omega} w \psi
\end{aligned}
$$

Suppose that $\tilde{\Phi} \in H^{2}(B)$ is a solution to the first minimization problem on the right hand side of (5.13). We have already proven that $\nabla \tilde{\Phi}$ solves (5.10), and taking the divergence therein shows that $\tilde{\Phi}$ solves the biharmonic equation $8 \Delta^{2} \Phi=-\Delta \varphi$ in $B$. As (f1) requires that $\Delta \varphi$ is not identically zero on $B$, we deduce that $\Delta \tilde{\Phi}$ is not identically zero as well. This implies by Young inequality

$$
\int_{B} 8 \tilde{\Phi}_{x y}^{2}+2\left(\tilde{\Phi}_{y y}-\tilde{\Phi}_{x x}\right)^{2}+\int_{B} \tilde{\Phi} \Delta \varphi<\int_{B} 8 \tilde{\Phi}_{x y}^{2}+4 \tilde{\Phi}_{x x}^{2}+4 \tilde{\Phi}_{y y}^{2}+\int_{B} \tilde{\Phi} \Delta \varphi .
$$

Notice that the inequality is strict, since the Young inequality $2\left(\tilde{\Phi}_{y y}-\tilde{\Phi}_{x x}\right)^{2} \leq 4 \tilde{\Phi}_{x x}^{2}+4 \tilde{\Phi}_{y y}^{2}$ holds with equality if and only if $\tilde{\Phi}_{x x}=-\tilde{\Phi}_{y y}$, and we have just checked that $\Delta \tilde{\Phi}$ does not vanish identically on $B$. In particular, $2\left(\tilde{\Phi}_{y y}-\tilde{\Phi}_{x x}\right)^{2}<4 \tilde{\Phi}_{x x}^{2}+4 \tilde{\Phi}_{y y}^{2}$ on a set of positive 
measure in $B$. From Lemma 5.1, from (5.14) and (5.13) we infer

$$
\begin{aligned}
& \min _{H^{1}\left(\Omega, \mathbb{R}^{3}\right)} \tilde{\mathcal{G}} \\
& \leq \min _{u \in H^{2}(B)} \int_{B} 8 u_{x y}^{2}+2\left(u_{y y}-u_{x x}\right)^{2}+\int_{B} u \Delta \varphi+\min _{w \in H^{1}(0,1)} 4 \pi \int_{0}^{1} w^{\prime 2}-\pi \int_{0}^{1} w \psi \\
& \leq \int_{B} 8 \tilde{\Phi}_{x y}^{2}+2\left(\tilde{\Phi}_{y y}-\tilde{\Phi}_{x x}\right)^{2}+\int_{B} \tilde{\Phi} \Delta \varphi+\min _{w \in H^{1}(0,1)} 4 \pi \int_{0}^{1} w^{\prime 2}-\pi \int_{0}^{1} w \psi \\
& <\int_{B} 8 \tilde{\Phi}_{x y}^{2}+4 \tilde{\Phi}_{x x}^{2}+4 \tilde{\Phi}_{y y}^{2}+\int_{B} \tilde{\Phi} \Delta \varphi+\min _{w \in H^{1}(0,1)} 4 \pi \int_{0}^{1} w^{\prime 2}-\pi \int_{0}^{1} w \psi \\
& =\min _{\Phi \in H^{2}(B)} \int_{B} 8 \Phi_{x y}^{2}+4 \Phi_{x x}^{2}+4 \Phi_{y y}^{2}+\int_{B} \Phi \Delta+\min _{w \in H^{1}(0,1)} 4 \pi \int_{0}^{1} w^{\prime 2}-\pi \int_{0}^{1} w \psi \\
& \leq \min _{H^{1}\left(\Omega, \mathbb{R}^{3}\right)} \mathcal{E} \leq \min _{H^{1}\left(\Omega, \mathbb{R}^{3}\right)} \mathcal{E}^{I}
\end{aligned}
$$

thus concluding the proof of the first statement.

Let us now prove the second statement, concerning the incompressible case. Let

$$
C(\varphi, \psi):=\|\psi\|_{L^{2}(\Omega)}\left(\int_{\Omega}|\nabla \varphi|^{2}+|\psi|^{2}\right)^{1 / 2} .
$$

If $\mathbf{v}^{*}=\left(v_{1}^{*}, v_{2}^{*}, v_{3}^{*}\right)$ minimizes $\mathcal{E}^{I}$ over $H^{1}\left(\Omega, \mathbb{R}^{3}\right)$, then we have

$$
0=\left.\frac{d}{d \varepsilon}\right|_{\varepsilon=0} \mathcal{E}^{I}\left((1+\varepsilon) \mathbf{v}^{*}\right)=8 \int_{\Omega}\left|\mathbb{E}\left(\mathbf{v}^{*}\right)\right|^{2}-\mathcal{L}\left(\mathbf{v}^{*}\right),
$$

and then by applying $(\mathcal{L} \mathbf{1})$ and Hölder inequality

$$
8 \int_{\Omega}\left|\mathbb{E}\left(\mathbf{v}^{*}\right)\right|^{2} d x=\mathcal{L}\left(\mathbf{v}^{*}\right)=\mathcal{L}\left(\mathbf{v}^{*}-\mathbb{P} \mathbf{v}^{*}\right) \leq\|\mathbf{f}\|_{L^{6 / 5}\left(\Omega, \mathbb{R}^{3}\right)}\left\|\mathbf{v}^{*}-\mathbb{P} \mathbf{v}^{*}\right\|_{L^{6}\left(\Omega, \mathbb{R}^{3}\right)} .
$$

By (3.5) and Hölder inequality again we deduce therefore

$$
\left(\int_{\Omega}\left|\mathbb{E}\left(\mathbf{v}^{*}\right)\right|^{2}\right)^{1 / 2} \leq K_{1}\|\mathbf{f}\|_{L^{2}\left(\Omega, \mathbb{R}^{3}\right)} \leq K_{1}\left(\int_{\Omega}|\nabla \varphi|^{2}+|\psi|^{2}\right)^{1 / 2}
$$

for some suitable constant $K_{1}$ (only depending on $\Omega$ ).

By taking into account (5.15), still by Korn and Hölder inequality and by (f2) we get

$$
\begin{aligned}
\left|\int_{\Omega} \psi v_{3}^{*}\right| & =\left|\int_{\Omega} \psi\left(\mathbf{v}^{*}-\mathbb{P} \mathbf{v}^{*}\right)_{3}\right| \leq\|\psi\|_{L^{2}(\Omega)}\left\|\mathbf{v}^{*}-\mathbb{P} \mathbf{v}^{*}\right\|_{L^{2}(\Omega)} \\
& \leq K_{2}\|\psi\|_{L^{2}(\Omega)}\left\|\mathbb{E}\left(\mathbf{v}^{*}\right)\right\|_{L^{2}(\Omega)} \leq K C(\varphi, \psi),
\end{aligned}
$$

where $K_{2}$ is another constant that depends only on $\Omega$ and $K=K_{1} K_{2}$. By taking into account (5.16), we get

$$
\min _{H^{1}\left(\Omega, \mathbb{R}^{3}\right)} \mathcal{E}^{I} \geq 4 \int_{\Omega}\left|v_{1, x}^{*}\right|^{2}+\left|v_{2, y}^{*}\right|^{2}+\frac{1}{2}\left(v_{1, y}^{*}+v_{2, x}^{*}\right)^{2}-\int_{\Omega}\left(v_{1}^{*} \varphi_{x}+v_{2}^{*} \varphi_{y}\right)-K C(\varphi, \psi)
$$

and by Jensen inequality

$$
\begin{aligned}
\min _{H^{1}\left(\Omega, \mathbb{R}^{3}\right)} \mathcal{E}^{I} & \geq 4 \int_{B}\left(\left|\tilde{v}_{1, x}^{*}\right|^{2}+\left|\tilde{v}_{2, y}^{*}\right|^{2}+\frac{1}{2}\left(\tilde{v}_{1, y}^{*}+\tilde{v}_{2, x}^{*}\right)^{2}-\int_{B}\left(\tilde{v}_{1}^{*} \varphi_{x}+\tilde{v}_{2}^{*} \varphi_{y}\right)-K C(\varphi, \psi)\right. \\
& =\mathcal{J}\left(\tilde{\mathbf{v}}^{*}\right)-K C(\varphi, \psi)
\end{aligned}
$$


where we have $\operatorname{set} \tilde{\mathbf{v}}^{*}(x, y):=\int_{0}^{1} \mathbf{v}^{*}(x, y, z) d z$ and where $\mathcal{J}$ is defined by (5.9). By repeating the argument used in the compressible case (and letting $\tilde{\Phi}$ be defined in the same way) we obtain

$$
\begin{aligned}
\min _{H^{1}\left(\Omega, \mathbb{R}^{3}\right)} \mathcal{E}^{I} & \left.\geq \min _{\Phi \in H^{2}(B)} \int_{B} 8 \Phi_{x y}^{2}+4 \Phi_{x x}^{2}+4 \Phi_{y y}^{2}+\int_{B} \Phi \Delta \varphi\right)-K C(\varphi, \psi) \\
& =\int_{B} 8 \tilde{\Phi}_{x y}^{2}+4 \tilde{\Phi}_{x x}^{2}+4 \tilde{\Phi}_{y y}^{2}+\int_{B} \tilde{\Phi} \Delta \varphi-K C(\varphi, \psi) \\
& =\int_{B} 8 \tilde{\Phi}_{x y}^{2}+2\left(\tilde{\Phi}_{y y}-\tilde{\Phi}_{x x}\right)^{2}+2|\Delta \tilde{\Phi}|^{2}+\int_{B} \tilde{\Phi} \Delta \varphi-K C(\varphi, \psi) .
\end{aligned}
$$

By (5.17) and by the second statement of Lemma 5.1 we deduce

$$
\min _{H^{1}\left(\Omega, \mathbb{R}^{3}\right)} \mathcal{E}^{I} \geq \int_{B} 2|\Delta \tilde{\Phi}|^{2}-K C(\varphi, \psi)+\min _{H^{1}\left(\Omega, \mathbb{R}^{3}\right)} \tilde{\mathcal{G}}^{I} .
$$

Since $\int_{B}|\Delta \tilde{\Phi}|^{2}>0$ as previously observed, we may choose $\|\psi\|_{L^{2}(\Omega)}$ so small that $K C(\varphi, \psi)<\int_{B} 2|\Delta \tilde{\Phi}|^{2}$ and deduce

$$
\min _{H^{1}\left(\Omega, \mathbb{R}^{3}\right)} \mathcal{E}^{I}>\min _{H^{1}\left(\Omega, \mathbb{R}^{3}\right)} \tilde{\mathcal{G}}^{I}
$$

thus completing the proof.

We conclude by providing some more properties for the compressible case that are directly deduced by refining the arguments in the proof of Theorem 2.7, under the further assumption $\int_{0}^{1} z \psi(z) d z>0$. First we check that minimizers of the limit functional $\mathcal{G}$ are not unique up to infinitesimal rigid displacements, in the sense that there are two minimizers whose difference is not an infinitesimal rigid displacement. In a second statement we obtain a solution of the problem $\min \mathcal{G}$, thus showing more explicitly the gap between $\min \mathcal{G}$ and $\min \mathcal{E}$.

Proposition 5.2 Assume (2.10), (2.11), (2.12), (f1), (f2) and $\int_{0}^{1} z \psi(z) d z>0$. If $\mathbf{u} \in$ $\operatorname{argmin}_{H^{1}\left(\Omega, \mathbb{R}^{3}\right)} \mathcal{G}$, then $\mathbf{u}$ is of the form $\mathbf{u}=\mathbf{w}^{0}+\mathbf{w}$, where $\mathbb{E}\left(\mathbf{w}^{0}\right) \equiv 0$ and $\mathbf{w}=\left(w_{1}, w_{2}, w_{3}\right)$ is such that $w_{1}, w_{2}$ do not depend on $z$ and $w_{3}$ does not depend on $x, y$. Moreover, minimizers of $\mathcal{G}$ over $H^{1}\left(\Omega, \mathbb{R}^{3}\right)$ are not unique up to infinitesimal rigid displacements.

Proof Suppose that $\mathbf{u}=\left(u_{1}, u_{2}, u_{3}\right) \in H^{1}\left(\Omega, \mathbb{R}^{3}\right)$ is a minimizer for functional $\mathcal{G}$ and let $\overline{\mathbf{u}}:=\left(\bar{u}_{1}, \bar{u}_{2}, \bar{u}_{3}\right)$, where

$$
\bar{u}_{1}:=\int_{0}^{1} u_{1}(x, y, z) d z, \quad \bar{u}_{2}:=\int_{0}^{1} u_{2}(x, y, z) d z, \quad \bar{u}_{3}:=\frac{1}{\pi} \int_{B} u_{3}(x, y, z) d x d y .
$$

Since $\int_{0}^{1} z \psi(z) d z>0$, the set $\mathcal{S}_{\mathcal{L}}^{0}$ is given by (5.3), and then it is immediate to check that, due to the specific form of $\mathbf{f}$ from (2.11), there holds

$$
\int_{\Omega} \mathbf{f} \cdot \mathbf{R} \mathbf{u} d \mathbf{x}=\int_{\Omega} \mathbf{f} \cdot \mathbf{R} \overline{\mathbf{u}} d \mathbf{x} \quad \forall \mathbf{R} \in \mathcal{S}_{\mathcal{L}}^{0} .
$$

Moreover, by applying Jensen inequality, similarly to the proof of Theorem 2.7, we obtain

$$
\begin{array}{ll}
\int_{\Omega} u_{1, x}^{2} \geq \int_{\Omega} \bar{u}_{1, x}^{2}, & \int_{\Omega} u_{2, y}^{2} \geq \int_{\Omega} \bar{u}_{2, y}^{2} \\
\int_{\Omega} u_{3, z}^{2} \geq \int_{\Omega} \bar{u}_{3, z}^{2}, & \int_{\Omega}\left(u_{1, y}+u_{2, x}\right)^{2} \geq \int_{\Omega}\left(\bar{u}_{1, y}+\bar{u}_{2, x}\right)^{2}
\end{array}
$$


so that

$$
\begin{aligned}
& 4 \int_{\Omega}|\mathbb{E}(\mathbf{u})|^{2} d \mathbf{x} \\
& \quad=\int_{\Omega}\left(4 u_{1, x}^{2}+4 u_{2, y}^{2}+4 u_{3, z}^{2}+\left(u_{1, y}+u_{2, x}\right)^{2}+\left(u_{1, z}+u_{3, x}\right)^{2}+\left(u_{2, z}+u_{3, y}\right)^{2}\right) \\
& \quad \geq \int_{\Omega}\left(4 \bar{u}_{1, x}^{2}+4 \bar{u}_{2, y}^{2}+4 \bar{u}_{3, z}^{2}+\left(\bar{u}_{1, y}+\bar{u}_{2, x}\right)^{2}\right)=4 \int_{\Omega}|\mathbb{E}(\overline{\mathbf{u}})|^{2} d \mathbf{x} .
\end{aligned}
$$

But the latter inequality and the inequalities (5.19) are necessarily equalities, otherwise in view of (5.18) we would deduce $\mathcal{G}(\overline{\mathbf{u}})<\mathcal{G}(\mathbf{u})$, contradicting minimality of $\mathbf{u}$. This implies

$$
u_{1, z}+u_{3, x}=u_{2, z}+u_{3, y} \equiv 0
$$

along with the fact that $u_{3, z}$ does not depend on $x, y$, since the Jensen inequality

$$
\frac{1}{\pi} \int_{B}\left(u_{3, z}\right)^{2} d x d y \geq\left(\frac{1}{\pi} \int_{B} u_{3, z} d x d y\right)^{2}=\bar{u}_{3, z}^{2}
$$

is strict unless $u_{3, z}$ is independent of $x, y$. Similarly, we deduce that $u_{1, x}, u_{2, y}$ and $u_{1, y}+u_{2, x}$ do not depend on $z$, therefore there exist functions $H=H(z), T=T(x, y), A=A(y, z)$, $B=B(x, y), C=C(x, z)$ and $D=D(x, y)$ such that $u_{1}, u_{2}, u_{3}$ have the form

$$
\begin{aligned}
& u_{1}(x, y, z)=A(y, z)+B(x, y), \\
& u_{2}(x, y, z)=C(x, z)+D(x, y), \\
& u_{3}(x, y, z)=H(z)+T(x, y),
\end{aligned}
$$

and there holds

$$
\partial_{z}\left(u_{1, y}+u_{2, x}\right)=A_{y z}(y, z)+C_{x z}(x, z) \equiv 0 .
$$

Taking (5.20) into account we deduce

$$
0 \equiv A_{z}(y, z)+T_{x}(x, y)=C_{z}(x, z)+T_{y}(x, y)
$$

so that $A_{z z}=C_{z z}=T_{x x}=T_{y y} \equiv 0$ and

$$
0 \equiv A_{y z}(y, z)+T_{x y}(x, y)=C_{x z}(x, z)+T_{x y}(x, y) .
$$

The latter entails, thanks to (5.22), $A_{y z}=C_{x z}=T_{x y} \equiv 0$. We conclude that $T$ is a linear function of $x, y$, i.e., $T(x, y)=a x+b y+c$ for some real constants $a, b, c$, and then from (5.23) we deduce that there are functions $Q=Q(y)$ and $S=S(x)$ such that $A(y, z)=-a z+Q(y)$ and $C(x, z)=-b x+S(x)$. Substituting in (5.21) we have

$$
\begin{aligned}
& u_{1}(x, y, z)=-a z+B(x, y)+Q(y), \\
& u_{2}(x, y, z)=-b z+D(x, y)+S(x), \\
& u_{3}(x, y, z)=H(z)+c+a x+b y,
\end{aligned}
$$

where $\mathbb{E}(-a z,-b z, c+a x+b y)=0$. This shows that if $\mathbf{u} \in H^{1}\left(\Omega, \mathbb{R}^{3}\right)$ minimizes $\mathcal{G}$, then up to adding an infinitesimal rigid displacement $u_{1}, u_{2}$ depend only on $x, y$ and $u_{3}$ depends only on $z$.

Let now $\mathbf{u}^{*}=\left(u_{1}^{*}, u_{2}^{*}, u_{3}^{*}\right) \in H^{1}(\Omega, \mathbb{R})^{3}$ be a minimizer of $\mathcal{G}$ such that $u_{1}^{*}, u_{2}^{*}$ do not depend on $z$ and $u_{3}^{*}$ does not depend on $x, y$, so that by defining $\hat{\mathbf{u}}:=\left(-u_{1}^{*},-u_{2}^{*}, u_{3}^{*}\right)$ we 
get $\left|\mathbb{E}\left(\mathbf{u}^{*}\right)\right|^{2} \equiv|\mathbb{E}(\hat{\mathbf{u}})|^{2}$. Let $\mathbf{R}^{*} \in \operatorname{argmax}_{\mathcal{S}_{\mathcal{L}}^{0}} \mathcal{L}\left(\mathbf{R u}^{*}\right)$. By letting $\hat{\mathbf{R}}:=\operatorname{diag}(-1,-1,1) \mathbf{R}^{*}$, we have $\hat{\mathbf{R}} \in \mathcal{S}_{\mathcal{L}}^{0}$ and we get

$$
\min _{H^{1}\left(\Omega, \mathbb{R}^{3}\right)} \mathcal{G}=\int_{\Omega}\left|\mathbb{E}\left(\mathbf{u}^{*}\right)\right|^{2} d \mathbf{x}-\mathcal{L}\left(\mathbf{R}^{*} \mathbf{u}^{*}\right)=\int_{\Omega}|\mathbb{E}(\hat{\mathbf{u}})|^{2} d \mathbf{x}-\mathcal{L}(\hat{\mathbf{R}} \hat{\mathbf{u}}),
$$

thus showing that $\hat{\mathbf{u}}$ is also a minimizer of $\mathcal{G}$. However, $\hat{\mathbf{u}}-\mathbf{u}^{*}$ is not an infinitesimal rigid displacement. Indeed, assume by contradiction that $\mathbb{E}\left(\mathbf{u}^{*}-\hat{\mathbf{u}}\right) \equiv 0$. Then $u_{1, x}^{*} \equiv u_{2, y}^{*} \equiv$ $u_{1, y}^{*}+u_{2, x}^{*} \equiv 0$, implying the existence of real constants $\bar{a}, \bar{b}, \bar{c}$ such that $u_{1}^{*}(x, y)=$ $\bar{a}+\bar{c} y$ and $u_{2}^{*}(x, y)=\bar{b}-\bar{c} x$. Therefore, $\left(0,0, u_{3}^{*}\right)$ differs from $\mathbf{u}^{*}$ by an infinitesimal rigid displacements, and since $\mathcal{G}$ is invariant under the addition of infinitesimal rigid displacements, we obtain the minimality of $\left(0,0, u_{3}^{*}\right)$ for $\mathcal{G}$. But then the form $(5.3)$ of $\mathcal{S}_{\mathcal{L}}^{0}$ and the fact that $u_{3}^{*}$ depends only on $z$ directly imply

$$
\min _{H^{1}\left(\Omega, \mathbb{R}^{3}\right)} \mathcal{G}=\mathcal{G}\left(0,0, u_{3}^{*}\right)=4 \int_{\Omega}\left(u_{3, z}^{*}\right)^{2} d \mathbf{x}
$$

so that $u_{3, z}^{*}$ needs to be identically zero and we deduce that the trivial displacement field minimizes $\mathcal{G}$, so that

$$
0=\mathcal{G}(\mathbf{0})=\min _{H^{1}\left(\Omega, \mathbb{R}^{3}\right)} \mathcal{G} \leq \min _{H^{1}\left(\Omega, \mathbb{R}^{3}\right)} \tilde{\mathcal{G}},
$$

where $\tilde{\mathcal{G}}$ is defined by (5.5). This contradicts Lemma 5.1 and concludes the proof.

In the next statement, for every $\theta \in[-\pi, \pi]$ and for $\mathbf{R}_{\theta}$ as in 5.3 , we use the notation

$$
\mathcal{G}_{\theta}(\mathbf{u})= \begin{cases}4 \int_{\Omega}|\mathbb{E}(\mathbf{u})|^{2} d \mathbf{x}-\mathcal{L}_{\mathbf{R}_{\theta}}(\mathbf{u}) & \text { if } \mathbf{u} \in H^{1}\left(\Omega, \mathbb{R}^{3}\right) \\ +\infty & \text { otherwise in } W^{1, p}\left(\Omega, \mathbb{R}^{3}\right),\end{cases}
$$

where $\mathcal{L}_{\mathbf{R}}$ is defined by (2.14). With this notation we clearly have $\mathcal{G}_{0} \equiv \mathcal{E}$ and $\mathcal{G}_{-\pi / 2} \equiv \tilde{\mathcal{G}}$, where $\tilde{\mathcal{G}}$ is defined by (5.5).

Proposition 5.3 Under the same assumptions of Proposition 5.2, let $\mathbf{u}_{0} \in \operatorname{argmin}_{W^{1, p}\left(\Omega, \mathbb{R}^{3}\right)} \mathcal{G}_{0}$ and let $\mathbf{u}_{-\pi / 2} \in \operatorname{argmin}_{W^{1, p}\left(\Omega, \mathbb{R}^{3}\right)} \mathcal{G}_{-\pi / 2}$. Then,

$$
\min _{W^{1, p}\left(\Omega, \mathbb{R}^{3}\right)} \mathcal{G}_{\theta}=\cos ^{2} \theta \mathcal{G}_{0}\left(\mathbf{u}_{0}\right)+\sin ^{2} \theta \mathcal{G}_{-\pi / 2}\left(\mathbf{u}_{-\pi / 2}\right)
$$

and

$$
\min _{W^{1, p}\left(\Omega, \mathbb{R}^{3}\right)} \mathcal{G}=\min _{\theta \in[-\pi, \pi]} \min _{\mathbf{u} \in W^{1, p}\left(\Omega, \mathbb{R}^{3}\right)} \mathcal{G}_{\theta}(\mathbf{u})=\mathcal{G}_{-\pi / 2}\left(\mathbf{u}_{-\pi / 2}\right)<\mathcal{G}_{0}\left(\mathbf{u}_{0}\right)=\min _{W^{1, p}\left(\Omega, \mathbb{R}^{3}\right)} \mathcal{E} .
$$

Proof It is possible to check with a computation that the vector field $2 r^{-1} \eta_{*}(r)(y,-x)$, where $r=\sqrt{x^{2}+y^{2}}$ and $\eta_{*}$ is defined by (5.12), solves the problem

$$
\begin{cases}-8 \operatorname{div} \widetilde{\mathbb{E}}(\tilde{\mathbf{u}})=\left(\varphi_{y},-\varphi_{x}\right) & \text { in } B \\ \widetilde{\mathbb{E}}(\tilde{\mathbf{u}}) \mathbf{n}=0 & \text { on } \partial B,\end{cases}
$$

where $\widetilde{\mathbb{E}}(\cdot)$ denotes the upper-left $2 \times 2$ submatrix of $\mathbb{E}(\cdot)$. Moreover, thanks to the very same argument of the proof of Proposition 5.2, it is possible to find a minimizer of functional 
$\mathcal{G}_{-\pi / 2}$ in which the first two components do not depend on $z$ and the third component does not depend on $x, y$. Therefore, it is possible to find such a minimizer by decoupling the corresponding Euler-Lagrange equation for $\mathbf{u}=\left(u_{1}, u_{2}, u_{3}\right)$, i.e.,

$$
\begin{cases}-8 \operatorname{div} \mathbb{E}(\mathbf{u})=\mathbf{R}_{\pi / 2}\left(\varphi_{x}, \varphi_{y}, \psi\right) & \text { in } \Omega \\ \mathbb{E}(\mathbf{u}) \mathbf{n}=0 & \text { on } \partial \Omega,\end{cases}
$$

in the above problem on $B$ for $\tilde{\mathbf{u}}=\left(u_{1}, u_{2}\right)$, and in the ordinary differential equation $-8 u_{3}^{\prime \prime}=\psi$ in the interval $(0,1)$, complemented by the conditions $u_{3}^{\prime}(0)=u_{3}^{\prime}(1)=0$, that gets solved, recalling (f2), by the function

$$
\Psi(z)=-\frac{1}{8} \int_{0}^{z} \int_{0}^{s} \psi(t) d t d s .
$$

Therefore, a minimizer of $\mathcal{G}_{-\pi / 2}$ over $W^{1, p}\left(\Omega, \mathbb{R}^{3}\right)$ is given by $\left(2 r^{-1} \eta_{*}(r) y,-2 r^{-1} \eta_{*}(r) x, \Psi(z)\right)$. Similarly, $\left(r^{-1} \eta_{*}(r) x, r^{-1} \eta_{*}(r) y, \Psi(z)\right)$ is a minimizer of $\mathcal{G}_{0} \equiv \mathcal{E}$, recalling that $r^{-1} \eta_{*}(r)(x, y)$ solves $(5.10)$ as seen in the proof of Theorem 2.7. Hence, given $\mathbf{u}_{0} \in$ $\operatorname{argmin}_{W^{1, p}\left(\Omega, \mathbb{R}^{3}\right)} \mathcal{G}_{0}$ and $\mathbf{u}_{-\pi / 2} \in \operatorname{argmin}_{W^{1, p}\left(\Omega, \mathbb{R}^{3}\right)} \mathcal{G}_{\pi / 2}$, we may assume w.l.o.g. that

$$
\mathbf{u}_{-\pi / 2}=\left(2 r^{-1} \eta_{*}(r) y,-2 r^{-1} \eta_{*}(r) x, \Psi(z)\right), \quad \mathbf{u}_{0}=\left(r^{-1} \eta_{*}(r) x, r^{-1} \eta_{*}(r) y, \Psi(z)\right),
$$

and more generally we let

$\mathbf{u}_{\theta}:=\cos \theta\left(r^{-1} \eta_{*}(r) x, r^{-1} \eta_{*}(r) y, 0\right)-\sin \theta\left(2 r^{-1} \eta_{*}(r) y,-2 r^{-1} \eta_{*}(r) x, 0\right)+(0,0, \Psi(z))$, so that $\mathbf{u}_{\theta}$ solves

$$
\begin{cases}-8 \operatorname{div} \mathbb{E}(\mathbf{u})=\mathbf{R}_{\theta}^{T}\left(\varphi_{x}, \varphi_{y}, \psi\right) & \text { in } \Omega \\ \mathbb{E}(\mathbf{u}) \mathbf{n}=0 & \text { on } \partial \Omega,\end{cases}
$$

for any $\theta \in[-\pi, \pi]$, and then $\mathbf{u}_{\theta}$ is indeed a minimizer of $\mathcal{G}_{\theta}$. Taking advantage of radiality and of the form of $\mathbf{u}_{\theta}$, it is easy to check that

$$
\int_{\Omega} \mathbb{E}\left(\mathbf{u}_{0}\right): \mathbb{E}\left(\mathbf{u}_{-\pi / 2}\right) d \mathbf{x}=0
$$

and that (5.24) holds. But as shown in the proof of Theorem 2.7 we have

$\mathcal{G}_{0}\left(\mathbf{u}_{0}\right)=\min _{W^{1, p}\left(\Omega, \mathbb{R}^{3}\right)} \mathcal{G}_{0}=\min _{W^{1, p}\left(\Omega, \mathbb{R}^{3}\right)} \mathcal{E}>\min _{W^{1, p}\left(\Omega, \mathbb{R}^{3}\right)} \tilde{\mathcal{G}}=\min _{W^{1, p}\left(\Omega, \mathbb{R}^{3}\right)} \mathcal{G}_{-\pi / 2}=\mathcal{G}_{-\pi / 2}\left(\mathbf{u}_{-\pi / 2}\right)$,

so that

$$
\min _{\theta \in[-\pi, \pi]} \mathcal{G}\left(\mathbf{u}_{\theta}\right)=\min _{\theta \in[-\pi, \pi]} \cos ^{2} \theta \mathcal{G}_{0}\left(\mathbf{u}_{0}\right)+\sin ^{2} \theta \mathcal{G}_{-\pi / 2}\left(\mathbf{u}_{-\pi / 2}\right)=\mathcal{G}_{-\pi / 2}\left(\mathbf{u}_{-\pi / 2}\right) .
$$

We conclude that the optimal rotation realizing the maximum in the definition of $\mathcal{G}\left(\mathbf{u}_{-\pi / 2}\right)$ is given by $\mathbf{R}_{-\pi / 2}$ and that (5.25) holds true.

We eventually remark that in view of the latter propositions (and in the same assumptions) and in view of Theorem 2.10, by taking $\theta= \pm \pi / 2$, there is no gap between the minimal value of functional $\mathcal{G}_{\mathbf{R}_{\theta}}$ and that of functional $\mathcal{E}_{\mathbf{R}_{\theta}}$.

Acknowledgements The authors acknowledge support from the MIUR-PRIN project No 2017TEXA3H. The authors are members of the GNAMPA group of the Istituto Nazionale di Alta Matematica (INdAM).

Funding Open access funding provided by Università degli Studi di Genova within the CRUI-CARE Agreement. 
Open Access This article is licensed under a Creative Commons Attribution 4.0 International License, which permits use, sharing, adaptation, distribution and reproduction in any medium or format, as long as you give appropriate credit to the original author(s) and the source, provide a link to the Creative Commons licence, and indicate if changes were made. The images or other third party material in this article are included in the article's Creative Commons licence, unless indicated otherwise in a credit line to the material. If material is not included in the article's Creative Commons licence and your intended use is not permitted by statutory regulation or exceeds the permitted use, you will need to obtain permission directly from the copyright holder. To view a copy of this licence, visit http://creativecommons.org/licenses/by/4.0/.

\section{References}

1. Agostiniani, V., Blass, T., Koumatos, K.: From nonlinear to linearized elasticity via Gamma-convergence: the case of multiwell energies satisfying weak coercivity conditions. Math. Models Methods Appl. Sci. 25(01), 1-38 (2015)

2. Agostiniani, V., Dal Maso, G., De Simone, A.: Linear elasticity obtained from finite elasticity by Gammaconvergence under weak coerciveness conditions. Ann. Inst. H. Poincaré Anal. non Linéaire 29, 715-735 (2012)

3. Alicandro, R., Dal Maso, G., Lazzaroni, G., Palombaro, M.: Derivation of a linearised elasticity model from singularly perturbed multiwell energy functionals. Arch. Ration. Mech. Anal. 230, 1-45 (2018)

4. Dal Maso, G., Negri, M., Percivale, D.: Linearized elasticity as $\Gamma$-limit of finite elasticity. Set-Valued Anal. 10(2-3), 165-183 (2002)

5. Friesecke, G., James, R.D., Müller, S.: A theorem on geometric rigidity and the derivation of non linear plate theory from three dimensional elasticity. Commun. Pure Appl. Math. 55, 1461-1506 (2002)

6. Friesecke, G., James, R.D., Müller, S.: A hierarchy of plate models from nonlinear elasticity by Gammaconvergence. Arch. Rational Mech. Anal. 1(80), 183-236 (2006)

7. Gurtin, M.E.: An Introduction to Continuum Mechanics. Academic Press, New York (1981)

8. Jesenko, M., Schmidt, B.: Geometric linearization of theories for incompressible elastic materials and applications. Math. Models Methods Appl. Sci. 31(4), 829-860 (2021)

9. Maddalena, F., Percivale, D., Tomarelli, F.: A new variational approach to linearization of traction problems in elasticity. J. Optim. Theory Appl. 182, 383-403 (2019)

10. Maddalena, F., Percivale, D., Tomarelli, F.: The gap in pure traction problems between linear elasticity and variational limit of finite elasticity. Arch. Ration. Mech. Anal. 234, 1091-1120 (2019)

11. Mainini, E., Percivale, D.: Linearization of elasticity models for incompressible materials, preprint, arXiv:2004.09286

12. Mainini, E., Percivale, D.: Variational linearization of pure traction problems in incompressible elasticity. Z. Angew. Math. Phys. 71, 146 (2020)

13. Maor, C., Mora, M.G.: Reference configurations vs. optimal rotations: a derivation of linear elasticity from finite elasticity for all traction forces. J. Nonlinear Sci. 31: 62 (2021)

14. Nitsche, J.A.: On Korn's second inequality. RAIRO Anal. Numér. 15, 237-248 (1981)

15. Schmidt, B.: Linear Gamma-limits of multiwell energies in nonlinear elasticity theory. Contin. Mech. Thermodyn. 20(6), 375-396 (2008)

Publisher's Note Springer Nature remains neutral with regard to jurisdictional claims in published maps and institutional affiliations. 